\title{
Digital Heritage as a Scholarly Field-Topics, Researchers, and Perspectives from a Bibliometric Point of View
}

\author{
SANDER MÜNSTER, Media Center/Digital Humanities, Technische Universität Dresden/ \\ Friedrich-Schiller-Universität Jena
}

Digital heritage comprises a broad variety of approaches and topics and involves researchers from multiple disciplines. Against this background, this article presents a four-stage investigation on standards, publications, disciplinary cultures, as well as scholars in the field of digital heritage and particularly tangible objects as monuments and sites, carried out in 2016 and 2017. It includes results of (1) the inquiry of nearly 4,000 publications from major conferences, (2) a workshop-based survey involving 44 researchers, (3) 15 qualitative interviews, as well as (4) two online surveys with 1,000 and 700 participants, respectively. As an overall finding, the community is driven by researchers from European countries, especially Italy, with a background in humanities. Cross-national co-authorships are promoted by cultural and spatial closeness and-probably due to funding policy-EU membership. A discourse is primarily driven by technologies, and the most common keywords refer to the technologies used. Most prominent research areas are data acquisition and management, visualization, and analysis. Recent topics are, for instance, unmanned airborne vehicle (UAV)-based 3D surveying technologies, augmented and virtual reality visualization, metadata and paradata standards for documentation, and virtual museums. Since a lack of money is named as the biggest obstacle nowadays, competency and human resources are most frequently named as demand. An epistemic culture in the scholarly field of digital heritage is closer to engineering than to humanities. Moreover, conference series are most relevant for a scientific discourse, and especially EU projects set pace as most important research endeavors.

CCS Concepts: • Applied computing $\rightarrow$ Arts and humanities; Digital libraries and archives; • Information systems $\rightarrow$ Information extraction; Link and co-citation analysis;

Additional Key Words and Phrases: Digital Heritage, survey, topics, scholarly field

\section{ACM Reference format:}

Sander Münster. 2019. Digital Heritage as a Scholarly Field-Topics, Researchers, and Perspectives from a Bibliometric Point of View. ACM J. Comput. Cult. Herit. 12, 3, Article 22 (July 2019), 27 pages.

https://doi.org/10.1145/3310012

\section{INTRODUCTION}

At a glance, digital heritage comprises a broad variety of approaches and topics and involves researchers from multiple disciplines. Vice versa, there are multiple concepts tackling both digital methods and cultural heritage. A main distinction is that between digital heritage and digital humanities. Cultural heritage objects are the research object of both digital humanities and digital heritage. The latter comprises digitally born and digitized

Most of the research that this article is based on was part of the junior research group UrbanHistory4D's activities, which has received funding from the German Federal Ministry of Education and Research under Grant Agreement No. 01UG1630. The survey on challenges and demands was done as part of Virtual Multimodal Museum (ViMM) Coordination and Support Action (CSA) activities, funded under the EU Horizon 2020 program under Grant Agreement No. 727107.

Authors’ address: S. Münster, 01069 Dresden/Fürstengraben 1, 07743 Jena; email: sander.muenster@tu-dresden.de.

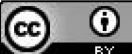

This work is licensed under a Creative Commons Attribution International 4.0 License.

(C) 2019 Copyright held by the owner/author(s).

1556-4673/2019/07-ART22

https://doi.org/10.1145/3310012

ACM Journal on Computing and Cultural Heritage, Vol. 12, No. 3, Article 22. Publication date: July 2019. 
Table 1. Digital Approaches to Cultural Heritage

\begin{tabular}{|c|c|c|}
\hline Digital Heritage & Digital Humanities & $\begin{array}{c}\text { Digital Discipline (e.g., } \\
\text { Archaeology) }\end{array}$ \\
\hline \multicolumn{3}{|c|}{$\begin{array}{l}\text { - Subject: cultural heritage } \\
\text { • Digital technologies } \\
\text { hnological and partly methodological challenges }\end{array}$} \\
\hline $\begin{array}{l}\text { - Research interest: Preserving, } \\
\text { researching, communicating a } \\
\text { specific heritage object } \\
\text { Disciplinary references: e.g., } \\
\text { archaeology, computer science, } \\
\text { museology, geo- and engineering } \\
\text { sciences }\end{array}$ & $\begin{array}{l}\text { - Investigation of questions in the } \\
\text { humanities with digital methods } \\
\text { - Disciplinary references: } \\
\text { humanities and computer science }\end{array}$ & $\begin{array}{l}\text { - Research and information } \\
\text { practices in a specific scholarly } \\
\text { field } \\
\text { - Disciplinary references: e.g., } \\
\text { archaeology, art and architectural } \\
\text { history, library and information } \\
\text { sciences (LIS) }\end{array}$ \\
\hline
\end{tabular}

"cultural, educational, scientific, and administrative resources, as well as technical, legal, medical, and other kinds of information" [1]. While digital humanities focus on the application of digital technologies to support research in the humanities (cf., e.g., References [2, 3, 4]), the scholarly community on cultural heritage concentrates on both, tangible and intangible cultural heritage and their preservation, education and research (cf. Reference [1]). Both concepts employ digital technologies often in cross-disciplinary settings, and they often use similar technologies [5] (Table 1). Despite communalities, there is only little overlap between related scholarly communities in terms of involved researchers and research associations. Digital humanities originated in language studies [6] with early and continued emphasis on text encoding and analysis [7]. However, in recent years a greater focus on images as well as historical artifacts, buildings, and sites got established (cf. Reference [8]). Additionally, various disciplines rose specific disciplinary approaches as, for instance, digital art history or digital archaeology. A common bond is their focus not only on research but also on information practices in the particular scholarly field [9].

With regards to this article's focus on digital heritage, some central topics include:

- Documentation (Geometric, Architectural, Historic, etc.), involving 2D and/or 3D for archiving, research, preservation, management, protection, and so on.

- Accurate measurements, suitable for restoration, reconstructions, structural studies, protection, and so on.

- Monitoring of its state, involving recording deformations, materials, assessing pathology, and so on.

- Proper Management of data for sustainability, risk management, accessibility, and so on.

- Preservation possibilities especially suited for fragile objects (e.g., libraries, etc.)

- Public Outreach, involving "visualization, dissemination, raising awareness of the public and many more" (cited according to Reference [10]).

Against this background, this article investigates current research topics, challenges and practices in the field of digital heritage. Due to the investigation being based on conference proceedings from large international conference series, this covers mainly the field of tangible heritage as monuments, sites and environments. Concrete questions are:

- What are research areas and topics?

- Who are researchers in that field?

- What are leading publications and projects?

\section{RESEARCH APPROACH}

\subsection{A characterization of a Scholarly Field}

What are the characteristics of a scholarly field? One prominent approach characterizes fields of research by specific epistemic cultures in terms of different "architectures of empirical approaches, specific constructions of 
the referent, particular ontologies of instruments, and different social machines" [11, p. 3], different approaches to gain insights, different vocabularies, different publication bodies and habits [12]. On a more operational level, disciplines are characterized to "(a) have a particular object of research [...], (b) have a body of accumulated specialist knowledge [...], (c) have theories and concepts [...], (d) use specific terminologies [...], (d) have developed specific research methods [...], and (e) must have some institutional manifestation in the form of subjects taught at universities or colleges [...]" [13]. From a practical point of view, the communities of practice approach originally introduced by Lave and Wenger [14] defines that these communities are marked by mutual engagement, a joint enterprise as well as a shared repertoire of knowledge and culture [15]. This latter definition seems most practical for this investigation to investigate (a) cooperation in terms of publication activities, (b) to investigate topics, methods and (c) cultures in terms of leading podia, projects as well as current issues and upcoming challenges.

\subsection{Digital Heritage Conferences and Publications}

An assumption is that publications such as conference papers and journals are main podia for knowledge sharing in academia. Over the past few decades, a scholarly community has become established around the subject of digital heritage, involving numerous researchers from different disciplines. This process has been accompanied by the appearance of various dedicated conferences, journals and books.

Comprehensively, this yielded the following starting point (cf. Reference [16]):

- On the one hand, in the field of history studies, no multidisciplinary, periodically held international conferences are known to deal specifically with digital technologies. On the other hand, numerous conferences and workshops include these topics. For instance, virtual museums are one of the topics of the International Council of Museums (ICOM) annual conference, just as the European Archaeological Association includes sections on digital methods.

- Conferences on digital technologies and approaches such as Sigraph or ACM conferences occasionally deal with digital heritage topics. Similarly, conferences on digital humanities as the Alliance of Digital Humanities Organizations (ADHO) annual meeting series are including some research on digital heritage.

- There are currently several major conferences specifically on digital aspects in the fields of archaeology and cultural heritage.

The International Workshop for 3D Virtual Reconstruction and Visualization of Complex Architectures (3DARCH), the Computer Applications and Quantitative Methods in Archaeology Conference (CAA), the symposium series organized by the Comité International de la Photogrammétrie Architecturale (CIPA) as a joint committee of the International Council on Monuments and Sites (ICOMOS) and the International Society for Photogrammetry and Remote Sensing (ISPRS), and the EUROMED conference series. The Digital Heritage Conference series covers a wide range of topics and scholarly communities. Conference series addressing certain aspects of cultural heritage and archaeology on an international level include the Eurographics Symposium on Graphics and Cultural Heritage (GCH), the International Conference on Cultural Heritage and New Technologies in Vienna (CHNT), the Conference on Virtual Systems and Multimedia (VSMM), and the CIDOC annual conferences. Two more important conferences have existed but were discontinued during the last years. These are the International Symposium on Virtual Reality, Archaeology and Cultural Heritage (VAST), and the Visualization in Archaeology workshop (VIA).

- For museums, the annual Museums and the Web conference series, the Electronic Visualisation and the Arts (EVA) series and the International Cultural Heritage Informatics Meetings (ICHIM; discontinued in 2007) are relevant podia. Also, some other conferences on museums in general contain sections about digital heritage. 
- The Journal of Cultural Heritage is an overarching organ for all digital humanities content. Several other journals dedicated to aspects of digital heritage have been founded over the last few years. Examples are the International Journal of Heritage in the Digital Era or the Journal for Digital Applications in Archaeology and Cultural Heritage.

In contrast to other scholarly fields, there are relatively few studies about publication output in the field of digital heritage. According to References [17] and [18], various factors can be identified that characterize humanities publication habits in contrast to other fields:

- Greater national and regional orientation, e.g., language-wise

- Fewer publications in series

- Less formalized forms of theoretical development

- More single researcher publications

- More publishing in non-scholarly publications

Moreover, "[...] formal scholarly communication covers only a part of D[igital] [Cultural] H[eritage]" [19]. This hypothesis has been empirically evaluated for archaeology [20], emphasizing that non-published literature is an important medium of scholarly knowledge generation and exchange. Extant studies applying bibliometrics to the humanities primarily focus on publication habits and their changes over time [21,22] or the appropriateness of bibliometric indexes in the humanities [17, 23, 24]. Some studies focus on topics and scholarly fields. Leydesdorff et al. mapped disciplinary areas and their relationships by analyzing citing patterns [23].

Consequently, the conferences studied and therefore the used questionnaires represent only a specific strandtangible cultural heritage such as historic environment, buildings, monuments and sites, including especially archaeological sites and remains. By and large, this excludes various other, mostly smaller communities. An important field that may not properly be represented is the study of intangible cultural heritage, such as rituals, fairy tales, tribal practices or crafts. Furthermore, only occasionally represented by the named conferences is the field of digital born cultural heritage comprising topics such as web archivalism or internet archaeology. ${ }^{1}$

With regards to a scholarly area of digital heritage and its adjacent field of digital humanities, Hicks et al. [18] stated that publication habits as well as research habits widely differ between single disciplines in the (digital) humanities. With regards to a scholarly community in digital humanities, Terras [25] examined that especially U.S.-, Canada-, and UK-based researchers contributed to an academic discourse up until 2006. While a community in Terras' analysis exclusively dealt with textual and-few-image sources, digital heritage related aspects such as visualization, geospatial analysis or VR/AR got into the top 50 keyword list in 2017 of Digital Humanities 2017 conference submissions [26]. A longitudinal study about digital humanities journal publications dating from 1989 to 2014 was performed by Tang et al. [7]. They investigated co-author and co-citation networks as well as topics by keywords. Concerning this latter point, they identified text mining, text analysis and geo information systems (GIS) as prominent topics for digital humanities.

With regards to digital heritage, Scollar [27] investigated the Conferences on Computer Application in Archaeologies (CAA) from 1971 to 1996, and the European Commission reports about projects done within the fifth to seventh framework programs [28]. Both studies found that researchers in the field of digital heritage are primarily located in Mediterranean countries and have backgrounds in various disciplines-including computing, humanities, architecture, and geo- and natural sciences. Concerning topics, Koutsabasis performed a literature-based survey about employed technologies and scenarios for interaction with cultural heritage [29]. According to his investigation, most prominent scenarios are virtual museums as well as on site presentations of cultural heritage. Beside these quantitative studies there are various qualitative discussions as, for instance, References [30,31].

\footnotetext{
${ }^{1}$ As example for publications in the field of internet archaeology: http://intarch.ac.uk/.
} 
Table 2. Overview of Investigational Stages

\begin{tabular}{|l|l|l|}
\hline Stage & \multicolumn{1}{|c|}{ Naming in text } & \multicolumn{1}{c|}{ Description } \\
\hline 1. Bibliometrics & Publication Sample A & $\begin{array}{l}\text { Bibliometric analysis of 452 publications in the field } \\
\text { of digital heritage mainly by manual classification } \\
\text { methods }\end{array}$ \\
\cline { 2 - 3 } & Publication Sample B & $\begin{array}{l}\text { Bibliometric analysis of an extended sample of 4,484 } \\
\text { publications in the field of digital heritage primarily } \\
\text { by automatized methods }\end{array}$ \\
\hline 2. In-conference survey & In-conference survey & $\begin{array}{l}\text { Questionnaire-based surveys at IFKAD and ARKDIS } \\
\text { conferences and at City University London in 2016 } \\
\text { with 44 researchers in total }\end{array}$ \\
\hline 4. Online Surveys & Online Survey A & $\begin{array}{l}\text { Qualitative Interviews with 15 researchers at several } \\
\text { universities in London in autumn 2016 }\end{array}$ \\
& $\begin{array}{l}\text { Online survey in spring 2017 on disciplines, topics, } \\
\text { methods, projects and publications with 988 } \\
\text { participants and 602 completed surveys }\end{array}$ \\
\cline { 2 - 3 } & Online Survey B & $\begin{array}{l}\text { Online survey in autumn 2017 on current challenges } \\
\text { and demands of stakeholders with 528 participants } \\
\text { and 349 completed surveys }\end{array}$ \\
\hline
\end{tabular}

\subsection{Topics and Methods in Digital Heritage}

The scholarly community on (digital) cultural heritage concentrates on tangible and intangible cultural heritage objects and their preservation, education and research [1]. What does it mean in practice? A general overview on cultural heritage as a research area in the first years of the millennium was described by the EPOCH project [32]. Particularly with regards to the use of digital methods in cultural heritage and digital humanities, Drucker [33] sketches a historical evolution as well as a current state of application of digital methods in humanities. Additionally, both Heusinger [34] and Kohle [35] define fields of supplement by digital tools and practices in research processes related to cultural heritage. Similarly, many texts describe a comprehensive state of the art and methodologies for digital archaeology (e.g., References [36-38]). Furthermore, there are many standards and guidelines as well as rules defined and discussed for dealing with historical content [39-43]. In contrast, there is little empirical research on practices and users of digital heritage [cf. 44].

\section{METHODOLOGY}

For the research questions presented in this article, I employed various methods from information sciences [45, 46]. As shown in Table 2, I performed four stages of investigation, including non-responsive investigations of publications and user research via surveys and interviews.

\subsection{Stage 1: Bibliometrics ${ }^{2}$}

The first stage was intended to identify relevant topics and trends and monitor their alteration over time by studying publications. Bibliometrics or-also widely used-informetrics [48] deals with the quantitative investigation of scientific structures and productivity by publications [49].

\footnotetext{
${ }^{2}$ Some of the results in this stage were originally published in: [16] Münster, S. and Ioannides, M. The scientific community of digital heritage in time and space. IEEE, Granada, 2015; [47] Münster, S. Employing bibliometric methods to identify a community, topics and protagonists of digital 3 D reconstruction in the humanities. Wuhan, 2017. Keywords employed (6/2012): digital heritage; 3D reconstruction; virtual architecture.
} 
Table 3. Samples (A: 452 Articles; B: 4,484 Articles)

\begin{tabular}{|c|c|c|c|c|}
\hline Publication & Volumes (Sample A) & No. of Articles & Volumes (Sample B) & No. of Articles \\
\hline 3DArch Conf. (bi-annual conf.) & $2005-2009$ & 112 & $2005-2017$ & 389 \\
\hline CAA Conf. (annual conf.) & 2007,2009 & 130 & $\begin{array}{c}1973-1992,1994-2001, \\
2004-2009,2011-2015\end{array}$ & 1,637 \\
\hline VAST Conf. (annual conf.) $^{4}$ & $2003-2007,2010$ & 105 & $2003-2006,2008-2012$ & 202 \\
\hline Digital Heritage (bi-annual conf.) $^{5}$ & & & 2013,2015 & 401 \\
\hline Euromed Conf. (bi-annual conf.) $^{5}$ & & & $2006-2016$ & 607 \\
\hline CIPA Conf. (bi-annual conf.) $^{6}$ & & 52 & $1999-2001,2005-2017$ & 1,248 \\
\hline Journal of Cultural Heritage $^{7}$ & $2000^{8}-2013$ & 53 & & \\
\hline $\begin{array}{c}\text { Various journal articles dealing } \\
\text { with Digital Heritage }\end{array}$ & $1999-2011$ & & & \\
\hline
\end{tabular}

Sample. At a glance, a key problem of all bibliometric research is the identification of relevant publications within a specific area. While standardized indexes like the Arts and Humanities Citation Index [50] or the European research Index in Humanities (ERIH) cover the whole range of disciplines, there are no indexes available for the particular field of digital heritage. Thus, the first task comprises index building for that field. Prior to creating a database, three experts were asked to identify relevant journals and conferences via semi-structured interviews. In addition, feedback from various discussions and presentations was included.

Data processing. These findings formed the basis for collecting the data samples presented in Table 3 . The first sample (Publication Sample A) included 452 publications and an extension included 4,484 publications (Publication Sample B). Relevant journal articles were identified by keyword search, and entire volumes of conference proceedings were included. The sample was restricted to articles written in English and available electronically, for practical reasons. The latter restriction meant that no publications of the VIA conference could be included, and several volumes of other conferences were left out. Moreover, all available contributions were included, regardless of length. In addition to conference papers, relevant articles from the Journal for Cultural Heritage, the Journal for Heritage in the Digital Era, and other periodicals were included. A major obstacle to building a research database was the fact that when it was compiled, many of the included conferences and journals were not listed in citation repositories or in publication databases like the ISI Web of Science, Scopus, or Google Scholar. This made it necessary to retrieve metadata from each single contribution via text analysis. For each article, the following metadata were obtained:

- Names and affiliations of contributing authors

- Names and addresses of affiliating institutions

- Source data (conference and publication name, year, type of document)

- Publication title

- Keywords

\footnotetext{
${ }^{3}$ Proceedings published online: http://proceedings.caaconference.org/.

${ }^{4}$ Contributions published by Eurographics: https://diglib.eg.org/handle/10.2312/1003. ${ }^{6}$ Proceedings available via IEEE: http://ieeexplore.ieee. org/xpl/mostRecentIssue.jsp?punumber=6729393 (2013); http://ieeexplore.ieee.org/xpl/mostRecentIssue.jsp?punumber=7406203 (2015).

${ }^{5}$ Contributions published in Springer LNCS Series.

${ }^{6}$ Contributions published as special issues of ISPRS Archives and ISPRS Annals: http://www.isprs.org/publications/Default.aspx.

${ }^{7}$ ISSN: $1296-2074$.

${ }^{8}$ Selection of important articles identified via keyword-based search. The selected keywords derived from three expert interviews performed prior to the investigation.
} 
In a second step, I performed a qualitative data analysis for the 452 publications included in Sample A manually. This was a two-stage inductive and deductive qualitative content analysis [51], leading inductively to a categorization scheme, which was applied to the data. This identified and classified the cited projects and topics:

- Cited projects (name, date, institutions, object)

- Topics

The third step was to retrieve the full texts of all included publications in Sample B, providing a basis for quantitative text analysis. The full texts were then searched for information about funding institutions as well as for projects and standards. Moreover, all acknowledgments and references were extracted from these publications by text retrieval and were segmented automatically to retrieve publication years, main authors, title for publication, as well as founding institution, grant number, and project title for acknowledgments.

\subsection{Stage 2: In-conference Survey ${ }^{9}$}

To get more information about research topics, podia, and standards used beside a description in publications, I started a series of questionnaire-based surveys. These took place during a track on Visual Knowledge Management at the International Forum for Knowledge Asset Management (IFKAD) and the ARKDIS conference on information in archaeology, both held in June 2016, as well as during a lecture held at City University London in the department of human computer interaction in October 2016. In total, 44 researchers participated. Since these podia represented very specific facets of digital heritage, these were done to gain a general overview on topics and standards of relevance.

\subsection{Stage 3: Qualitative Interviews}

An adjacent interest was to investigate research topics, methods, and scholarly culture more in detail. I interviewed 15 researchers at several universities in London between September and November 2016, via structured expert interviews $[53,54]$. All interviews were recorded and semi-automatically transcribed by using the Pop Up Archive service in a non-public way. ${ }^{10}$ To analyze the interview transcripts, a qualitative content analysis approach was employed [55] to (1) inductively gain an initial category scheme and (2) apply it to further materials. Outcomes of that step comprise an inductively generated categorization scheme as well as a set of related variables and occurrences. Since a more extensive report of the investigation can be found in Reference [8], only selected results are referred to in this article to discuss and qualify the other, more data-driven findings.

\subsection{Stage 4: Online Surveys}

Since various phenomena were identified via these investigations, a follow-up interest was to investigate these issues among a larger number of researchers. Thus, another survey included all authors listed in the publications already mentioned for stage 1. I extracted all provided email addresses published under the author information section-in total 4,211 addresses. Against this background, several factors were limiting

1. Only recent publications contain email addresses of authors.

2. Often only the email address of the first author is included.

3. Nearly $1 / 4$ of the email addresses $(1,063$ of 4,211$)$ do not exist anymore.

Finally, I retrieved 3,148 valid email addresses that I used for two surveys. Both surveys used open questions only, to allow for diverse answers and to retrieve additional items [56].

\footnotetext{
${ }^{9}$ Some of the results from Stage 2 to 4 were originally published in: [52] Münster, S. A Survey on Topics, Researchers and Cultures in the Field of Digital Heritage. ISPRS Annals of the Photogrammetry, Remote Sensing and Spatial Information Sciences, IV-2/W2 (2017), $157-162$.

${ }^{10} \mathrm{https} / /$ archive.org/details/popuparchive, The Pop-Up Archive automated transcription service was discontinued in 2017.
} 


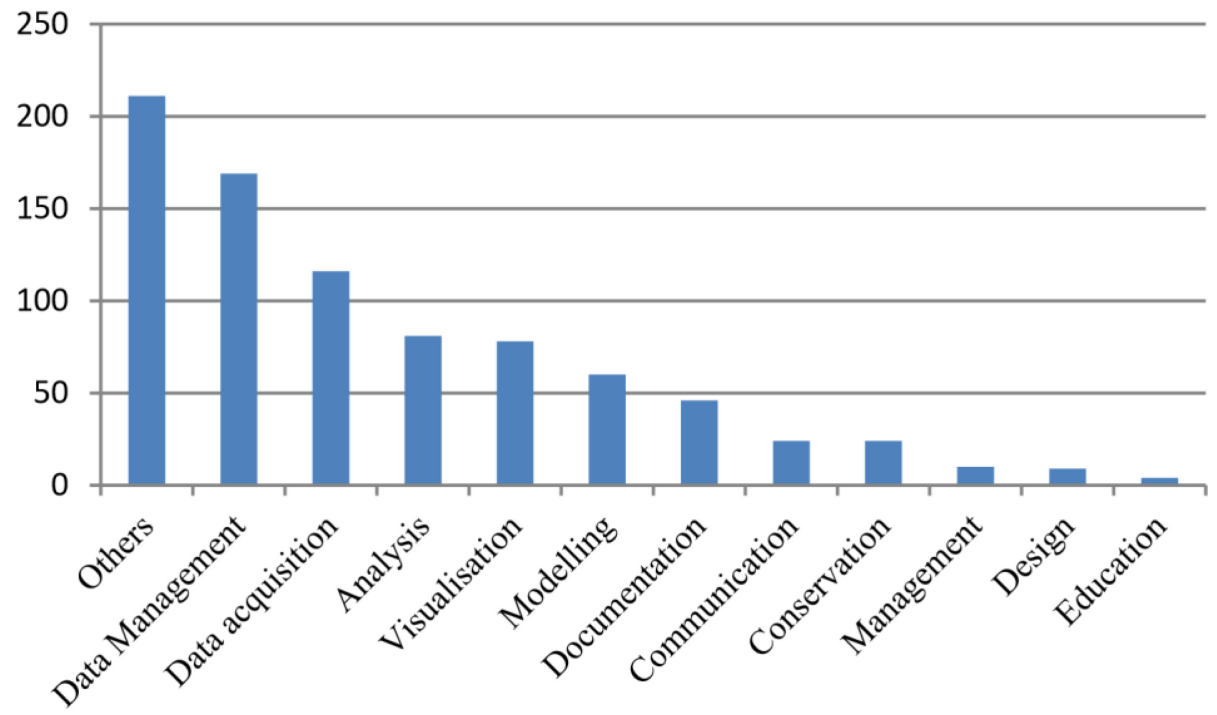

Fig. 1. Topics (Online Survey A, $\mathrm{n}=825$, categorization according to qualitative content analysis).

Online Survey A: A first survey took place over two weeks in March 2017 and contained questions on disciplines, topics, methods used by the participants as well as queries about their opinion on leading projects and publication bodies. 988 people participated and 602 completed the survey. Since the questions did not depend on each other, responses from non-finishers were included in the data analysis, too. The data analysis was based on the category scheme developed during the qualitative interviews and performed via inductive and deductive content analysis.

Online Survey B: Another interest was to know about current challenges and demands of stakeholders in the field of Digital Heritage. To investigate, I launched another online survey in August 2017, containing three questions asking for challenges, demands and most important institutions, again without any anchor examples. By November 15, 2017, 528 individuals had participated and 349 questionnaires were fully completed. ${ }^{11}$

\section{RESULTS AND DISCUSSION}

\subsection{Topics}

What are research areas and topics? A first objective was to identify the scope of topics comprised in the scholarly discussion on digital heritage. Concerning the relevance of individual topics (cf. Figure 1), especially data management was most frequently named, ranging from GIS and BIM to metadata schemes and data architecture (cf. Reference [52]). It was followed by data acquisition, photogrammetry, laser scanning, and other surveying technologies. Also mentioned were analysis, comprising, for instance, deep learning or simulation and visualization, including technologies such as virtual and augmented reality. As an additional note on the survey, a large number of answers did not fit into the predefined categories and were subsumed in "Others"-in most cases disciplinary approaches, epochs or objects were named.

How does this contrast to publications? To examine, I performed topic mining by an automated analysis of full texts. As a preparatory step I isolated nouns and reduced them to stems according to [58]. Topic extraction by factor analysis per paragraph employed Provalis WordStat [59]. Out of the resultant set of 20 topics, I identified

\footnotetext{
${ }^{11}$ Results were originally published in: [57] Münster, S., Ioannides, M. and Davies, R. International stakeholder survey on demands in the field of digital cultural heritage. 2017.
} 
Table 4. Topic Map Retrieved by Factor Analysis

\begin{tabular}{|c|c|c|l|l|}
\hline Rank & CASES & $\begin{array}{c}\text { PERCENTAGE } \\
\text { OF CASES }\end{array}$ & \multicolumn{1}{|c|}{ NAME } & \multicolumn{1}{|c|}{ KEYWORDS } \\
\hline 1 & 4,189 & $93,42 \%$ & Remote Sensing & Remote Sensing; Photogrammetry; Spatial \\
\hline 2 & 3,925 & $87,53 \%$ & Archaeology & Archaeology; Computing; CAA; Application \\
\hline 3 & 3,903 & $87,04 \%$ & Presentation & $\begin{array}{l}\text { Virtual; Interaction; Reality; Visitor; Exhibit; } \\
\text { Environment; VR; Experience; Game; Museum; } \\
\text { User }\end{array}$ \\
\hline 4 & 3,865 & $86,20 \%$ & Modelling & $\begin{array}{l}\text { Point; Coordinate; Distance; Camera; Measure; } \\
\text { Position; Control; Calibration; Accuracy; } \\
\text { Parameter; Error; Orientation }\end{array}$ \\
\hline 5 & 3,850 & $85,86 \%$ & Cultural Heritage; Conservation & Heritage; Culture; Conservation; Preservation \\
\hline 6 & 3,819 & $85,17 \%$ & Century; City & Century; City; Wall; Period; Roman; House \\
\hline 7 & 3,803 & $84,81 \%$ & Architecture; Building & $\begin{array}{l}\text { Architecture; Build; Element; BIM; Construction; } \\
\text { Geometry; Structure }\end{array}$ \\
\hline 8 & 3,695 & $82,40 \%$ & Visualization & $\begin{array}{l}\text { Texture; Resolution; Lighting; Render; Image; } \\
\text { Colour; Surface }\end{array}$ \\
\hline 9 & 3,485 & $77,72 \%$ & Data Management and Access & $\begin{array}{l}\text { User; Database; Web; Interface; File; Access; } \\
\text { Query }\end{array}$ \\
\hline 10 & 3,161 & $70,50 \%$ & Geo Approaches & GIS; Geography; Landscape; Map \\
\hline 11 & 3,035 & $67,69 \%$ & Automatic Algorithm & $\begin{array}{l}\text { Algorithm; Match; Extract; Automation; } \\
\text { Segment; Detect }\end{array}$ \\
\hline 12 & 2,484 & $55,40 \%$ & Photo-based Approaches & Photography; Aerial; Photogrammetry; Photo \\
\hline 13 & 2,081 & $46,41 \%$ & Laser Scanning & Laser; Scanner; Scanning; Cloud \\
\hline 14 & 1,509 & $33,65 \%$ & Sensor and Devices & Sensor; Device \\
\hline 15 & 939 & $20,94 \%$ & Ontologies & Ontologies; Semantic; CRM; Metadata \\
\hline
\end{tabular}

$\mathrm{n}=4,484$, Publication Sample B, "Cases": no. of publications included, 15 out of 20 retrieved topics, keywords were not named manually but identified via factor analysis.

15-manually named-topic sets (Table 4). A further five sets were excluded due to limited semantic coherencethese sets subsumed, for instance, nationalities or web-related terms as www and http.

Similar to the survey results presented below, topics are tightly related to the technologies and the disciplines of cultural heritage preservation and archaeology. While nearly all publications mention remote sensing technologies, particular approaches such as laser scanning and photogrammetry are named in half of the articles. Presentation and visualization issues like virtual reality and user-centred design are mentioned by $87 \%$ of the articles, also aspects of rendering and visualization, which are included in $82 \%$ of articles. In contrast to survey results, data management and access aspects such as databases and interfaces are slightly less frequently mentioned-in total by $3 / 4$ of the articles. Aspects of documentation and data linking like ontologies and semantics are mentioned by $21 \%$ of articles only.

Nevertheless, there is a vivid community specialized in these latter topics. In addition, content was frequently mentioned. With regards to that aspect, archaeology and computing are frequently referred to. Also, conservation and cultural heritage aspects are mentioned in $85 \%$ of the articles. Built heritage as well as related technologies such as BIM are another important topic, also referred by $85 \%$ of articles. Geobased content and technologies like GIS are also frequently mentioned-by $70 \%$ of the articles in total.

Since a majority of articles include multiple topics, this may indicate a high level of cross-topic cooperation. Furthermore, topics such as remote sensing, presentation and data management can be traced as very important in both individual research topics and publication content. Disciplinary links exist to computing, archaeology, geosciences, and preservation. Similarly, architecture and landscapes are the most important contents, which 


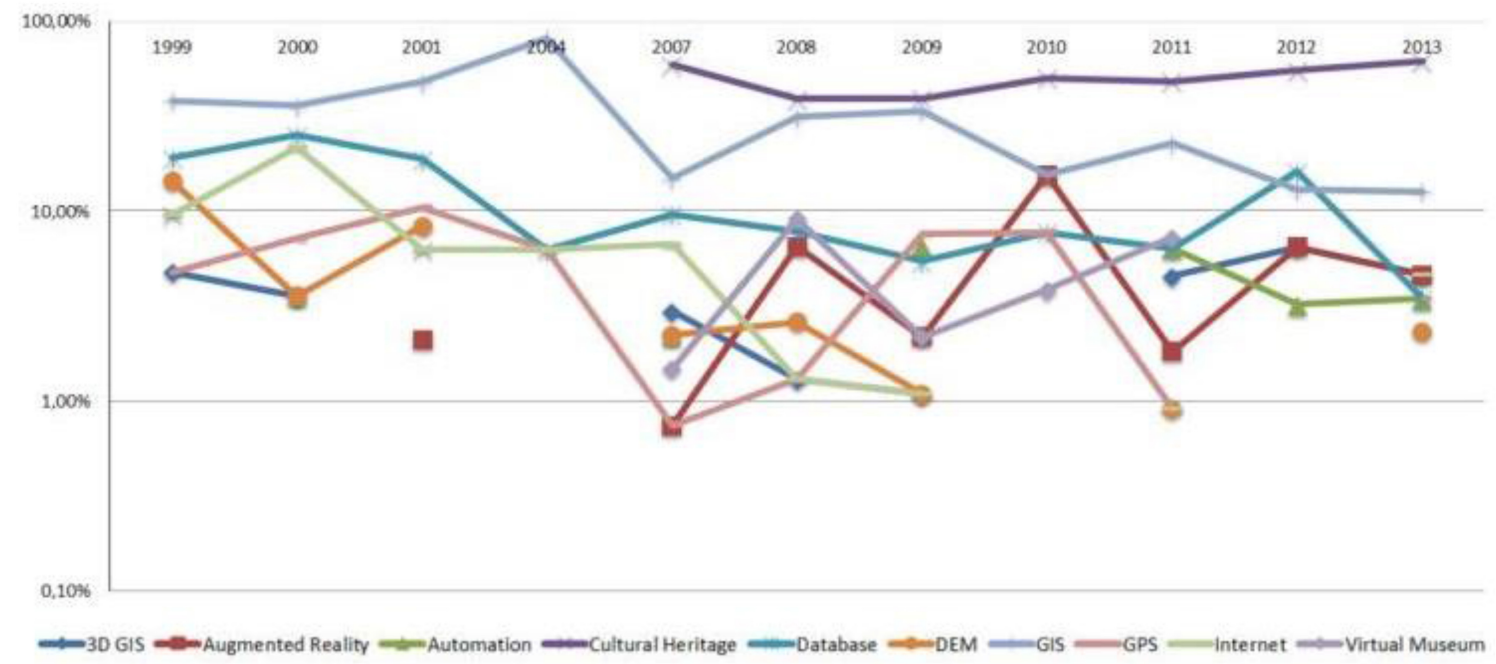

Fig. 2. Occurrences of selected keywords per year (in percentage of total keywords per year within all articles, graph exponentially scaled due to the different spread within single categories), Publication Sample B.

matches findings from former investigations [60]. Since there is a high congruence in topics retrieved by text analysis and survey, occasionally named topics such as design and education can be traced via survey only. Vice versa, via topic mining, some topics of general relevance such as automation and sensors became evident, which were not specifically mentioned in survey results.

Trends. Which trends are visible in publications? Two approaches to topic analysis were of interest here. The first was mapping the varying importance of research topics in recent years. For this longitudinal identification of trends, I considered the number of mentions of keywords in publications of a single cohort in relation to the total number of entries for that keyword. To avoid bias due to small samples, I included exclusively commonly used keywords $(\mathrm{n}>20)$ and years with more than 50 keyword entries. As shown in Figure 2, the importance of several topics changed over time. The rate of usage differs between topics. While some keywords, like cultural heritage and GIS, are used in the majority of articles, a small number of publications only names other keywords such as augmented reality. Besides numerous other less specific keywords mentioned (e.g., modelling or visualization) in the first period from 1999 to 2007, keywords such as database, GPS, internet, or DEM (digital elevation models) were frequently used. While database, DEM, and internet lost importance or were replaced by more sophisticated terms like web or 3D-GIS around the mid-2000s, GPS is still relevant. Since 2005, the topics virtual museums, virtual reality, close range sensing, laser scanning, and education have arisen. From 2008 to 2010, the research community has focused on technologies like LIDAR and augmented reality, and elaborated concepts for metadata and automation. Not all conferences have published their papers for the latter years yet, but these are expected to include upcoming trends such as 3D printing and Unmanned Aerial Vehicle (UAV)-based sensing.

What can we learn from the analysis about the research field of digital heritage? The community discourse is primarily about technologies and workflows, and new technologies are adopted early. For example, augmented reality, UAV, or Lidar were discussed at conferences and employed in projects shortly after availability as readyto-use technologies. As the automated text analysis identified, issues like documentation, accuracy, and semantics have been widely discussed in the community for many years. This corresponds to the results of a qualitative survey of various expert meetings and conferences to identify current challenges in the field of digital heritage [61], which named the development of scientific transparency, standards, and sustainable strategies to link data 


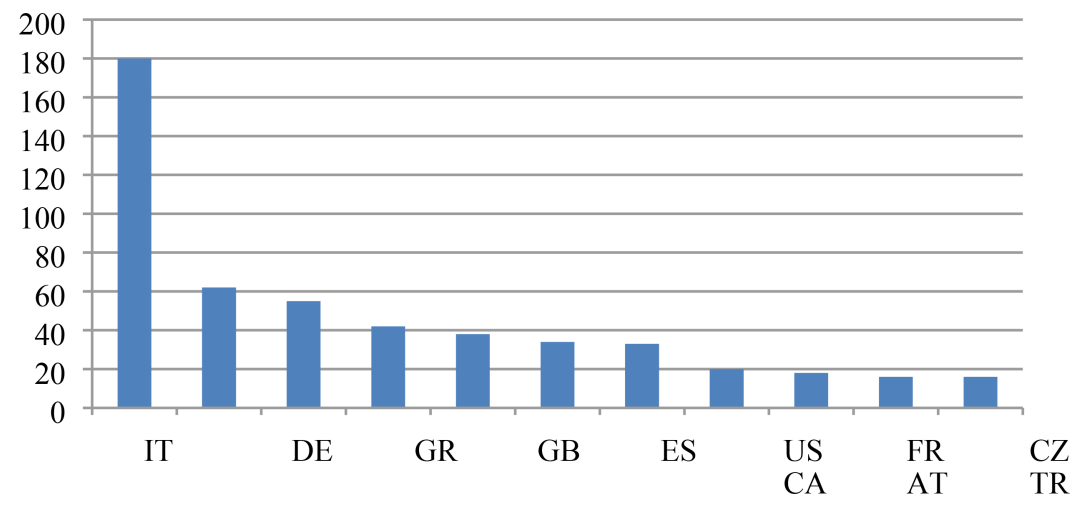

Fig. 3. Nationality of scholars in the field of digital heritage (Online Survey A, Top 10 out of $n=693$ ).

as still unresolved major challenges. Similarly, a majority of interviewees in the qualitative interview series estimated the access to data as big issue of digital humanities. That includes, for instance, aspects of data availability that is limited by legal barriers or company ownership. Since "much data is being shared [via] services like Facebook," it is-as mentioned by a research coordinator-"[potentially] going to be locked away and inaccessible" for researchers. Moreover, concerning aspects of long-term preservation and availability "we can't rely on commercial companies to pay [attention] for this." Furthermore, and beside the vast amount of data that is not available online for various reasons, much data is currently not accessible due to insufficient tagging, indexing or linking. Therefore, "we don't really know what's in there, if $[\ldots]$ a web page [links to] [...] broken images."

\subsection{Scholars}

What is the background of people who are active in the field of cultural heritage? Despite various attempts to attract researchers from other parts of the world, e.g., at conference locations in non-European countries, the community is primarily European. According to results from former studies [16, 28], also in the survey presented in this article the majority of researchers in the field of digital heritage are Italian, followed by Germans and Greek (cf. Figure 3). What are disciplinary backgrounds of authors? To classify research areas, I followed the 2007 edition of the Frascati manual offered by OECD [62]. Other schemes are rather outdated, such as CERIF [63], with resultant weaknesses esp. with regards to the distinction of computing areas or the limited compatibility to a European situation-such as the American NCES scheme [64]. To make this research comparable to former investigations, I grouped (1) all geo-related disciplines, i.e., cartography, surveying and geodesy, and (2) all architectural disciplines.

Concerning findings shown in Figure 4, a majority of participants assorted themselves to humanities. Most frequently named within this discipline was archaeology (cf. Reference [52]). These findings correspond to outcomes of the article-based survey in Section 4.1, but stood in contrast to former investigations, where especially the rate of computing was much higher [65]. It may be assumed that especially engineers and architectswhose rate is significant higher in this survey than in previous literature-based investigations-either (1) often work in departments dedicated to computing or (2)-since each authorship was counted in the literature-based assignment-have a lower publication output than researchers in computing.

Another assumption is that collaborative publications establish knowledge communication between authors. The basic idea is that in most cases, common authorship involves a personal connection and interaction between all the authors. Sociological role theory suggests that such a connection-whether weak or strong-could foster sharing and exchange of ideas and information [66]. Regarding structure, connections between people across disciplinary and national borders play an especially important role in disseminating information in social 


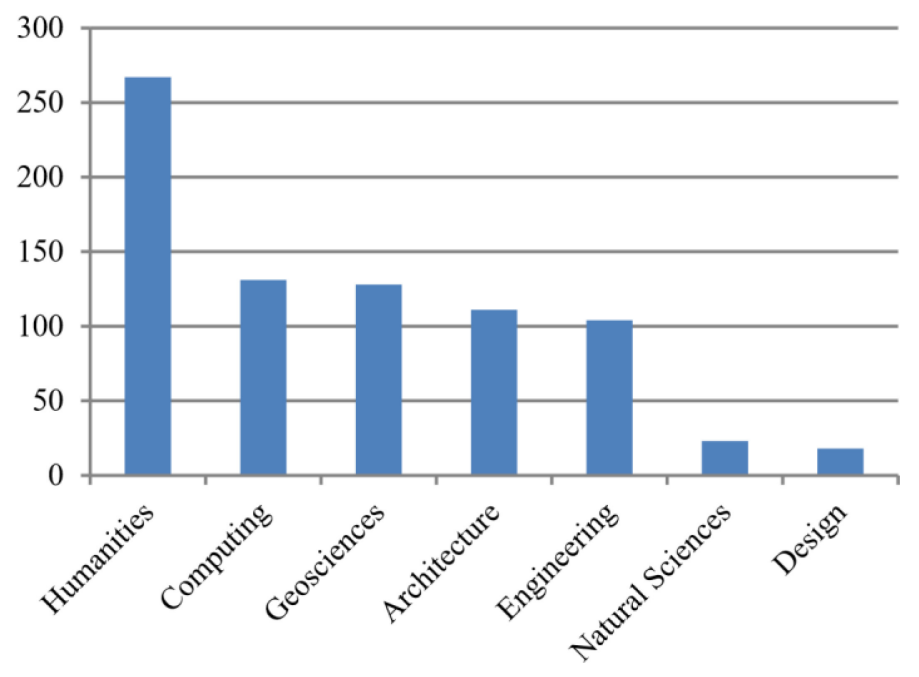

Fig. 4. Disciplinary background (Online Survey A, $n=782$ ).

communities. Nevertheless, information transfer in the context of joint publications is just assumed and it is impossible to reconstruct the intensity or even an existence of information transfer between authors based on empirical data (cf. Reference [67]). The publications in Bibliometric Sample B were written by 4,894 people and contain nearly 14,500 links between authors of cooperative articles. As shown in Figure 5, most were written by authors belonging to institutes in the same country (light blue). Persons in the top 10 in the categories of number of connections to other authors (degree), relevance as a connecting factor between author groups (betweenness centrality), or the number of publications (count), were highlighted in the graph (cf. Reference [68]). The giant component cluster (orange rectangle) contains 1,664 out of 2,951 (56\%) authors connected to each other. In comparison, the values for authors of the top ranked paper in the field of chemistry are over $90 \%[69,70]$, while values in digital humanities are below 20\% [7]. This indicates that there are more sub-communities in the field of digital heritage than in nature sciences, but a lower fragmentation-e.g., in terms of national or topic specific sub-communities-as in the digital humanities.

As a general finding, the people in the top 10 are especially highly connected internationally. To verify, I compared rates of international publications for these facilitators with those of the other authors. As shown in Table 5 , the rate of international publications involving facilitators is $28 \%$ compared to the rate of $21 \%$ of other authors.

I also discussed the results with various experts during conference workshops, e.g., at Digital Heritage 2015 or CAA 2013. As a qualitative response, the identified disseminators are generally not only active publishers but also often key players in the community in other ways, as members of scientific committees, conference chairs, initiators or leaders of projects. Regarding network structure, several clusters can be identified in the graph shown in Figure 5. For instance, Yakar and Yildiz (blue rectangle) are part of a relatively closely connected group of researchers at Turkish universities, characterized by a relatively large number of publication investments but only sparse international connections. This corresponds to the results from another investigation, on preferences for cross-national authorships [71]. While that investigation included three different types of linkage-crossnational authorship, citation of foreign publications, and citations by foreigners-this investigation focuses only on cross-national authorship due to the available data. First, an international co-authorship matrix is constructed as a $\mathrm{k} \times \mathrm{k}$ matrix, "where the element in the ith row, jth column is the number of papers with joint occurrence 


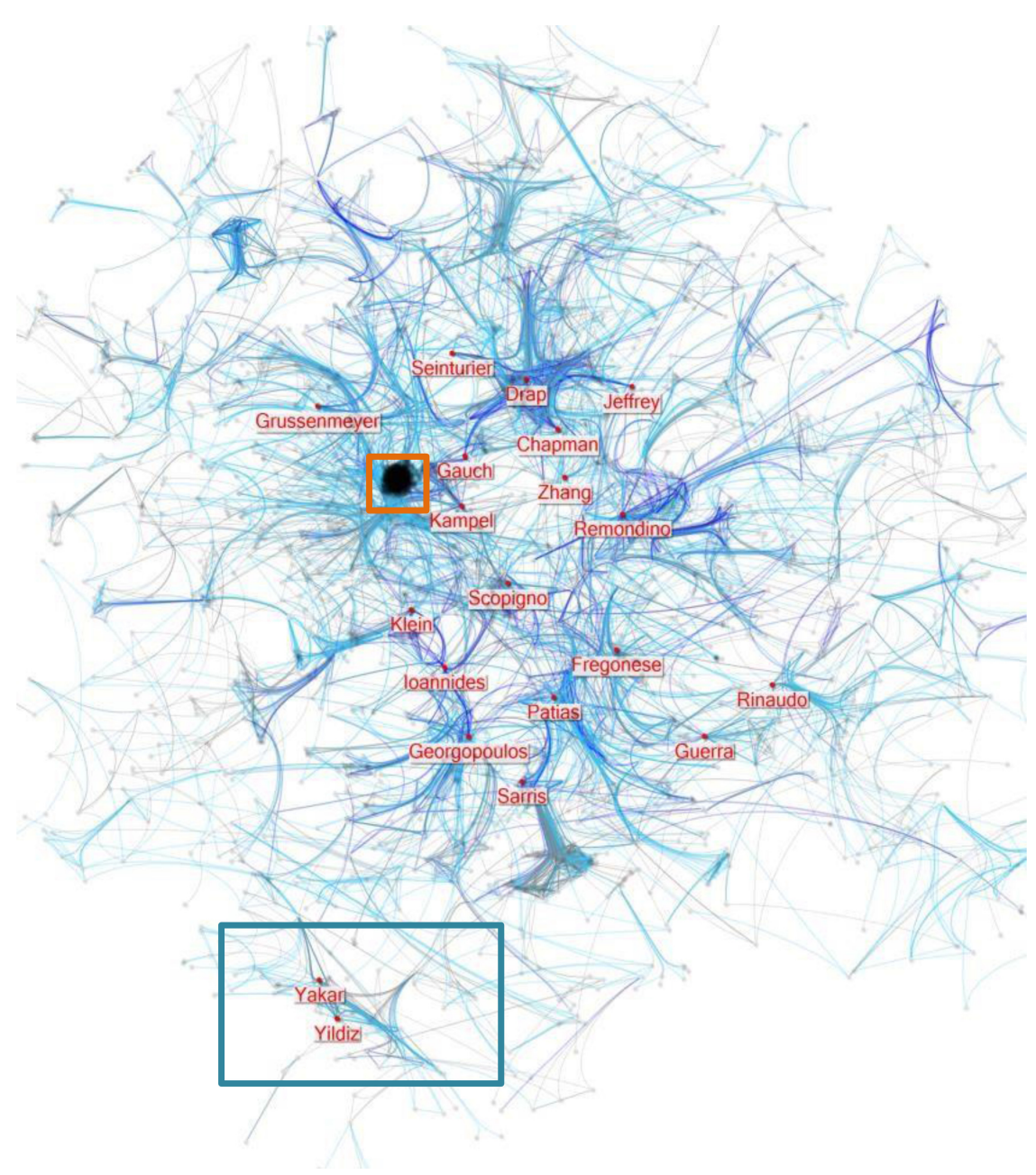

Fig. 5. Author-Co-author Relations (Top 10 in Degree, Count, Betweenness Centrality highlighted, Publication Sample B).

Table 5. Rate of International Publications (Publication Sample B)

\begin{tabular}{|c|c|c|}
\hline & Facilitators & Others \\
\hline Int. Publ. & $28.40 \%$ & $21.01 \%$ \\
\hline Nat. Publ. & $71.60 \%$ & $78.99 \%$ \\
\hline
\end{tabular}

of country $i$ and country $j$ in the byline of the paper. Evidently, since co-authorship has no direction, the coauthorship matrix is symmetric" [71, p. 410].

According to Schubert and Glänzel, the inclusion of countries, from which just a few articles were published, would lead to an overrepresentation of these countries and falsify the results. Therefore, I included only countries with at least 100 author-author relations. Since I was interested in the cooperation between researchers as individuals, I counted each relationship between persons involved in a single publication. Consequently, publica- 
Table 6. Preferred Cross-National Cooperation Partners, Publication Sample B

\begin{tabular}{|c|c|c|c|c|c|}
\hline \multirow{2}{*}{ Country } & \multirow{2}{*}{ No. } & \multirow{2}{*}{ Int. Publ. Percent } & \multicolumn{3}{|c|}{ Preferred Cooperation } \\
\cline { 4 - 6 } & & & $\mathbf{1 s t}$ & 2nd & 3rd \\
\hline AT & 631 & $44.7 \%$ & DE & BE & GB \\
\hline BE & 510 & $39.0 \%$ & GB & AT & CH \\
\hline BR & 192 & $20.3 \%$ & DE & FR & ES \\
\hline CA & 283 & $41.7 \%$ & IT & US & DE \\
\hline CH & 235 & $62.1 \%$ & IT & BE & DE \\
\hline CN & 123 & $9.8 \%$ & IT & NL & US \\
\hline CY & 269 & $58.4 \%$ & GR & GB & IT \\
\hline CZ & 154 & $11.7 \%$ & IT & DE/US & \\
\hline DE & 1,293 & $25.9 \%$ & AT & GR & IN / IT \\
\hline ES & 904 & $8.6 \%$ & IT & GB & FR \\
\hline FI & 133 & $5.3 \%$ & US/GB & & FR \\
\hline FR & 1,587 & $35.9 \%$ & IT & PT & GB \\
\hline GB & 1,158 & $40.0 \%$ & FR & IT & BE \\
\hline GR & 1,707 & $15.9 \%$ & CY & DE & GB \\
\hline IN & 112 & $30.4 \%$ & DE & AT & GB \\
\hline IT & 3,469 & $20.2 \%$ & FR & US & GB \\
\hline JP & 454 & $12.6 \%$ & DE & US & AT \\
\hline NL & 109 & $47.7 \%$ & IT & GB/GR & \\
\hline PT & 411 & $55.5 \%$ & FR & IT & GB \\
\hline RU & 101 & $5.0 \%$ & BE & & \\
\hline TR & 587 & $5.8 \%$ & DE & GR & US \\
\hline US & 934 & $27.4 \%$ & IT & GB & CA \\
\hline
\end{tabular}

tions written by large author collectives led to more occurrences than those by smaller groups. Unlike Schubert and Glänzel, I also included domestic publications. Otherwise, due the calculation method, countries with just a limited number of publications would be extremely overrepresented in the results. The indicator to reveal a cross-national "affinity" has been described in several publications (e.g., Reference [72]). According to Glänzel, it follows this formula:

$$
\mathrm{QA}(\mathbf{i}, \mathbf{j})=(\mathrm{XA}(\mathbf{i}, \mathbf{j}) / \mathrm{XA}(\mathbf{i}, *)) /(\mathbf{X A}(*, \mathbf{j}) /(\mathrm{XA}(*, *)-\mathrm{XA}(\mathbf{i}, *))),
$$

where $\mathrm{XA}(\mathrm{i}, \mathrm{j})$ defines an element in the $i$ th row as well as $j$ th column of a co-authorship matrix. $\mathrm{XA}(\mathrm{i}, *)$ and $\mathrm{XA}(*, \mathrm{j})$ define row sums of the $i$ th as well as $j$ th row. $\mathrm{XA}(*, *)$ defines the sum of the overall matrix. When the sum of the rows and columns are included in the calculations, the result is highly dependent on the total number of domestic and cross-national publications of both corresponding nations. Factors that foster cooperation between authors of two nations include cultural closeness in terms of common language, spatial closeness, or largescale research facilities [73]. Another factor is that most EU funding programs require cooperating partners from at least three countries. This may explain why EU countries prefer to collaborate with other EU countries (cf. Table 6), and why spatial or lingual proximity is less important in these cases. The proportion of cross-national publications ranges from $5 \%$ to over $60 \%$.

Frequency of contribution. One of the most common indicators is the number of publications per author. The sample shown in Figure 6 correlates well with Lotka's distribution function, which covers a wide range of disciplines and their publications [74]. The distribution curve shows that a very small number of authors with 
No. of Authors

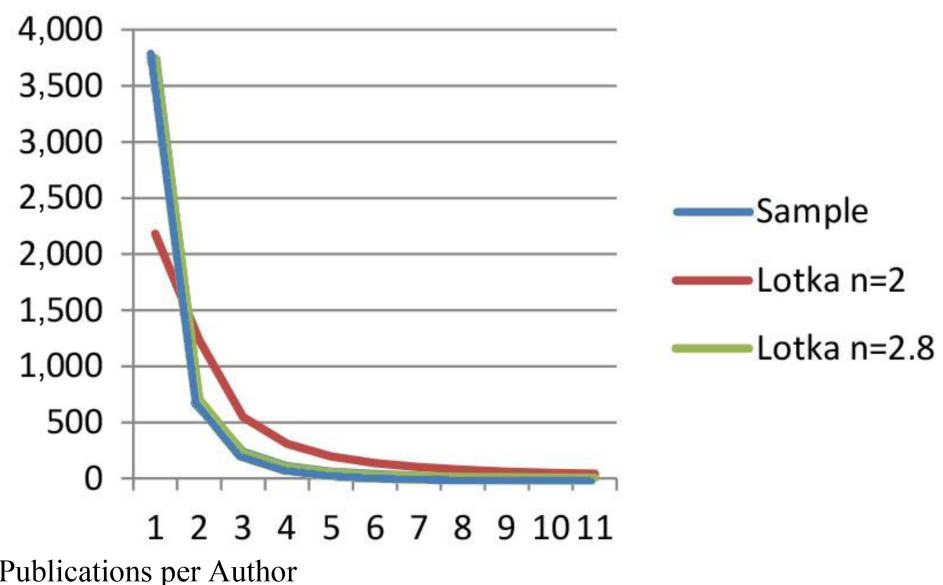

Fig. 6. Frequency distribution curves ( $n=4,894$ authors, Publication Sample B).

multiple publications contrasts a large number of authors with only one publication. This follows the formula

$$
\mathrm{Y}=\mathrm{C} / \mathrm{X}^{\mathrm{n}}
$$

where $\mathrm{C}$ is the total number of authors included $(\mathrm{n}=4,894)$ and $\mathrm{X}$ indicates the number of publications of each cohort (authors with $1,2, \ldots$ publications). The exponent $\mathrm{n}$ is a constant. Lotka postulated an average exponent of $\mathrm{n}=2$, which varies significantly by discipline $[49,75,76]$. More recent studies assumed an average value of 2.3 to 2.5 [77]. The empirical findings for the distribution function of the investigated publications coincide with $\mathrm{n}=2.8$. Any further interpretation of these values must be estimated based on a relatively small and potentially flawed sample. Compared to the values cited in the literature, the above-average value for this investigation indicates low publication productivity, with a disproportionate number of authors who are only occasionally involved.

Another aim was to map for how long researchers are involved in the community. As shown in Figure 7, most of the researchers contributing frequently to the investigated publications started their involvement in the early 2000s and contribute nearly on an annual basis. Moreover, for the period from 1990 it is visible that currently a second generation of researchers is active and many of those persons active in the 1990s have retired by now. ${ }^{12}$

\subsection{Institutions}

Besides the view on prolific individuals, it is interesting to know which institutions are the most relevant. Therefore, all publications authored by researchers from an institution were aggregated. Concerning an outcome, only European research facilities and primarily universities are amongst the Top 10 in the categories of Degree, Betweenness Centrality, or the count of publications (Figure 8).

An adjacent question is how much single researchers influence the relevance of an institution. Taking both the top ranked institutions and researchers into concern (Table 7), one finding is that the best ranked authors were contributing to an average of $45 \%$ of the institutions' publications. Also, there are stable affiliations-in average authors are writing $80 \%$ of their articles for the same institution. Generally spoken, the visibility of an institution highly depends on single researchers and only few institutions as, for instance, the University of Florence, the

\footnotetext{
${ }^{12}$ It has to be mentioned that some of the conference series started before the $1990 \mathrm{~s}$.
} 


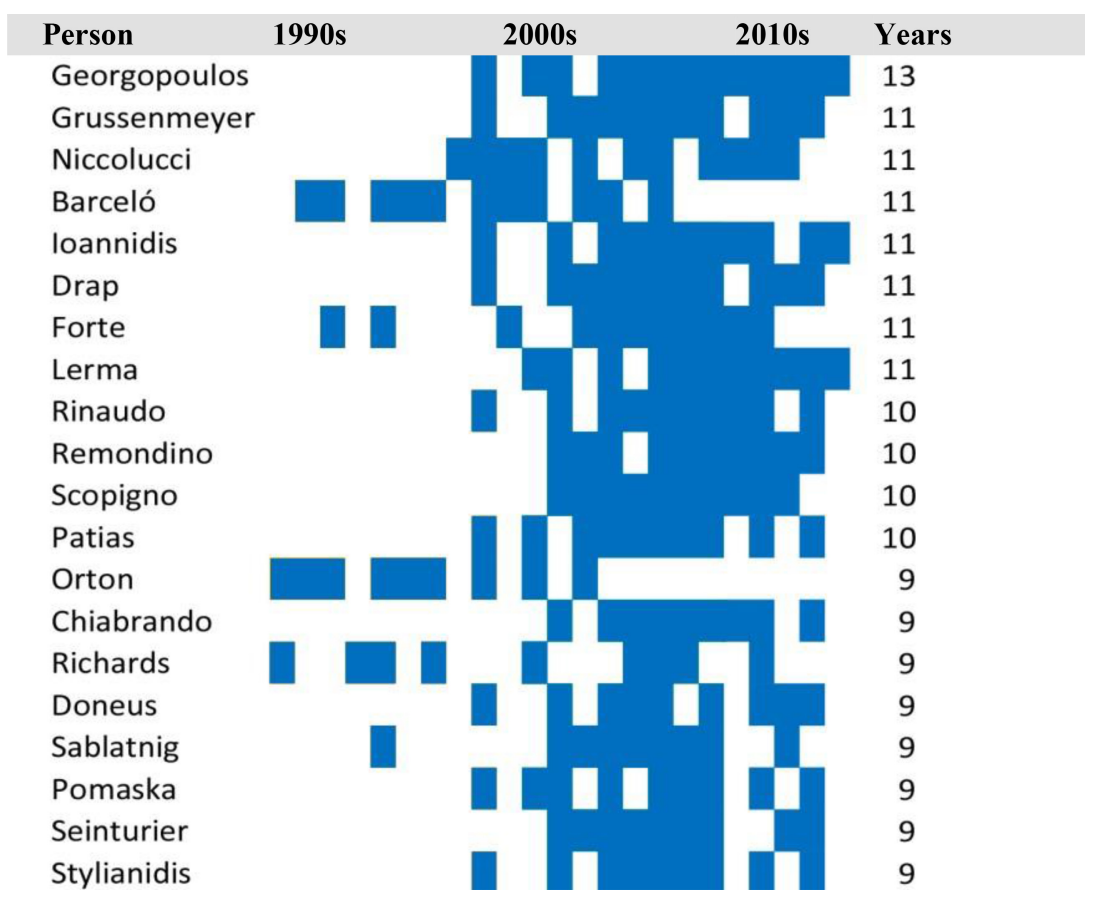

Fig. 7. Most frequently active persons (years active $>8$, Publication Sample B).

KU Leuven or the Politechnico di Milano have established multiple independent researchers or teams within this domain.

Which institutions do researchers assess as "world leading"? Within our online survey, we asked for world leading institutions in the field of cultural heritage and virtual museums. Concerning a response, eight of the institutions amongst the top 20 are museums and collections, five of them are located in the UK or USA. Most frequently named were the British Museum and the Getty foundation (Figure 9). Seven institutions amongst the top 20 are associations, of which most frequently named were worldwide heritage institutions such as UNESCO and ICOMOS, ICOM, ICCROM or CIPA. On a national level, especially English Heritage and CNRS are well renowned. Three universities are amongst the top 20-UCL, the University of York and the Cyprus University of Technology. In addition, one company-Google at \#13-was named. Several participants would see no world leading institution (\#8). The bias between survey results and publication-based research may be caused by querying explicitly for cultural heritage and museums in the survey as well as due to the conferences as specifically academic way of scholarly communication. Consequently, European research institutions are highly active in publishing at the initially mentioned major scientific conferences while American and English associations are more prestigious and renowned in the field of cultural heritage and museums.

\subsection{An epistemic View}

What are standards in digital heritage $?^{13}$ Much discussion in the field of digital heritage concerns standards. Queried within the in-conference workshops in stage 2, not only publications and projects (which were named as

\footnotetext{
${ }^{13}$ This paragraph was originally published in Reference [52] Münster, S. A Survey on Topics, Researchers and Cultures in the Field of Digital Heritage. ISPRS Annals of the Photogrammetry, Remote Sensing and Spatial Information Sciences, IV-2/W2 (2017), 157-162.
} 


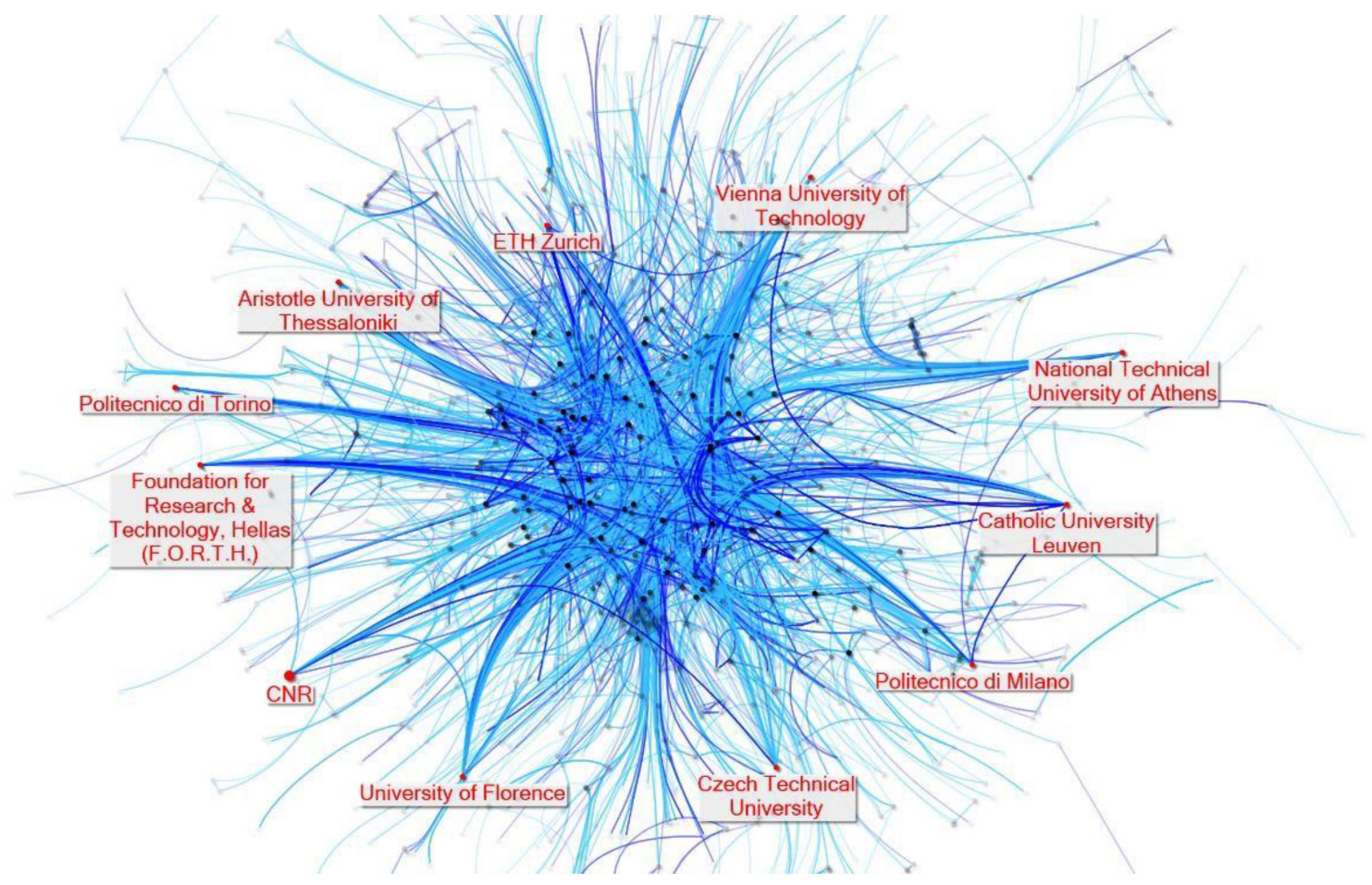

Fig. 8. Author-Co-Author Relations-Institutions (Top 10 in Degree, Betweenness Centrality highlighted, Publication Sample B).

anchor examples in the questionnaire) were named as "standards" but also various data repositories and services (cf. Table 8), which underlines the high relevance of data as well as the availability of high-quality infrastructures.

Concerning publications, both conference series and journals were named nearly equally often, but no books or book series were mentioned. Moreover, "methods"-even if named as anchor example-were named only occasionally. An explanation may be that digital heritage is - as mentioned by a head of digital museum technologies"around the subject," incorporating a wide plurality of contributing institutions as well as methods and approaches. While many single projects were named as influencing in the online survey, only a few were mentioned by multiple researchers. Similarly, there is probably no single institution or method explicitly mentioned as standard-maybe due to the "diverse nature of the methods used" [78, p. 48]. To verify these findings empirically, I searched for some of the most relevant standards in the publication sample B. Specifically, I searched for the London and Seville Charter, which define the visualization of cultural heritage, the ICOMOS charter, which defines frameworks for heritage conservation, and CIDOC-CRM, which defines a widely accepted metadata standard for cultural heritage (Table 9). It has to be mentioned that sometimes these standards are younger than the publications in the sample used for this article. Also, the Getty Art and Architecture Thesaurus (AAT) and the Cultural Objects Name Authority (CONA) ontologies were searched. Nevertheless, these standards are only occasionally cited in publications, too. A weak indication-due to the randomly selected keywords as well as a missing comparison to other scientific areas-may be that these standards do not have that practical relevance for a whole community.

What are most relevant publication bodies? Against that background, I queried within the online survey which podia were estimated as most relevant. Since I selected the sample on the base of specific conference series 
Table 7. Importance of Single Researchers in Institutions (Best Ranked Researchers Per Institution Named, Publication Sample B)

\begin{tabular}{|c|c|c|c|c|c|}
\hline Name & $\begin{array}{l}\text { Publications } \\
\quad \text { (total) }\end{array}$ & $\begin{array}{l}\text { Publications } \\
\text { (at named } \\
\text { Institution) }\end{array}$ & Institution & $\begin{array}{l}\text { Publications } \\
\text { (total) }\end{array}$ & Quota \\
\hline Niccolucci & 19 & 9 & University of Florence & 67 & $13 \%$ \\
\hline Kamermans & 6 & 6 & Catholic University Leuven & 40 & $15 \%$ \\
\hline Tucci & 18 & 11 & University of Florence & 67 & $16 \%$ \\
\hline Fregonese & 14 & 13 & Politecnico di Milano & 68 & $19 \%$ \\
\hline Seinturier & 13 & 5 & Ecole d'Architecture de Marseille & 20 & $25 \%$ \\
\hline Kampel & 14 & 14 & Vienna University of Technology & 56 & $25 \%$ \\
\hline Drap & 23 & 8 & Ecole d'Architecture de Marseille & 26 & $31 \%$ \\
\hline Remondino & 31 & 10 & ETH Zurich & 31 & $32 \%$ \\
\hline Rinaudo & 31 & 31 & Politecnico di Torino & 78 & $40 \%$ \\
\hline Georgopoulos & 35 & 27 & National Technical University of Athens & 61 & $44 \%$ \\
\hline Pavelka & 18 & 17 & Czech Technical University & 37 & $46 \%$ \\
\hline Patias & 23 & 23 & Aristotle University of Thessaloniki & 47 & $49 \%$ \\
\hline Sarris & 20 & 16 & $\begin{array}{l}\text { Foundation for Research and } \\
\text { Technology Hellas (F.O.R.T.H.) }\end{array}$ & 32 & $50 \%$ \\
\hline Yildiz & 19 & 18 & Selcuk University & 35 & $51 \%$ \\
\hline Fangi & 16 & 15 & Polytechnic University of Marche & 29 & $52 \%$ \\
\hline Scopigno & 22 & 22 & CNR & 42 & $52 \%$ \\
\hline Yakar & 20 & 20 & Selcuk University & 35 & $57 \%$ \\
\hline Fellner & 17 & 12 & Graz University of Technology & 18 & $67 \%$ \\
\hline Guerra & 20 & 15 & University Iuav of Venezia & 21 & $71 \%$ \\
\hline Lerma & 16 & 15 & Polytechnic University of Valencia & 21 & $71 \%$ \\
\hline Grussenmeyer & 30 & 24 & Strasbourg University & 30 & $80 \%$ \\
\hline Chapman & 14 & 8 & University of Hull & 9 & $89 \%$ \\
\hline
\end{tabular}

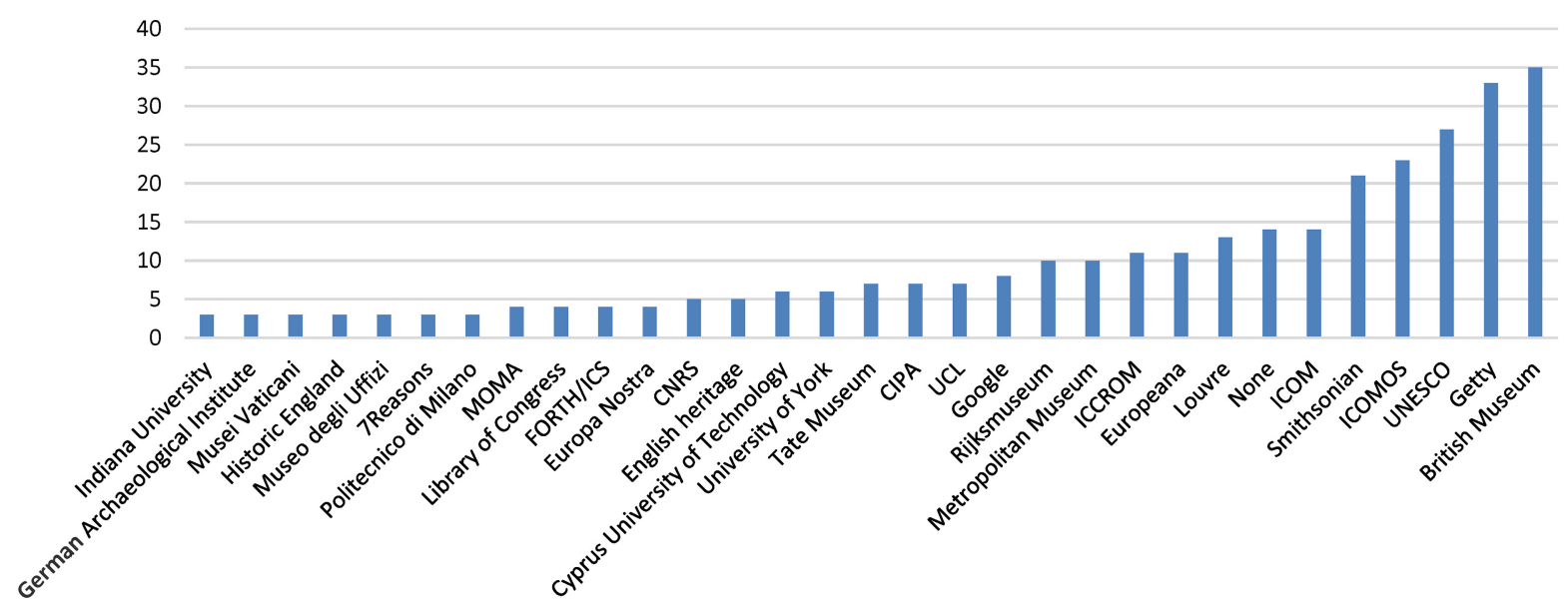

Fig. 9. Who do you consider to be world leading institutions in the field of cultural heritage and museums? (Online Survey $\mathrm{B}, \mathrm{n}=648$ named items, $\mathrm{n}>2$ ). 
Table 8. Answers to the Question "What are 'Gold Standards' in your Field of Research?"

(In-conference Survey,

21 Contributors, 56 Answers)

\begin{tabular}{|c|c|}
\hline Item & Count \\
\hline Publications & 20 \\
Repositories & 12 \\
Projects & 8 \\
Institutions & 5 \\
Standards & 4 \\
not recognizable & 4 \\
Methods & 2 \\
Others & 1 \\
\hline
\end{tabular}

Table 9. Citation of Selected Standards in Publications ( $n=4,484$ Publications, Publication Sample B)

\begin{tabular}{|l|c|}
\hline Search term & No. of Articles references \\
\hline Seville Charter & 31 \\
\hline London Charter & 101 \\
\hline ICOMOS Charter ${ }^{14}$ & 171 \\
\hline CIDOC-CRM & 148 \\
\hline Getty Art and Architecture Thesaurus (AAT) & 30 \\
\hline Getty Cultural Objects Name Authority (CONA) & 36 \\
\hline
\end{tabular}

as shown in sect. 3.1, I assumed that results would be biased especially with regards to named conferences. Nevertheless, I expected (1) to query further podia and especially journals of relevance as well as (2) to derive a ranking of publications.

Concerning conference series named, especially the CAA series was mentioned as most relevant (Figure 10), followed by the relatively young Digital Heritage series that took place in 2013 for the first time. Among journals (Figure 9), those published by the ISPRS were mentioned as most relevant, especially the ISPRS archives and annals. A major influencing factor for that may be that all proceedings of CIPA symposia and workshops were published in special issues of these journals. Furthermore, the ACM Journal on Computing and Cultural Heritage (JOCCH) and the Journal of Archaeological Science were named as relevant (Figure 11). Since conference series were named nearly twice as often as journals, it may be assumed that communication in the field of digital heritage primarily takes place via conferences. In total, 531 different publication bodies were named-undoubtedly there exists a large variety of podia of relevance for researchers in the field of digital heritage.

Projects. Another question in the online survey A was about projects that are estimated as most groundbreaking. Similar to publications, a wide variety of projects was named as most relevant (cf. Figure 12). Concerning these references, all top 10 ranked projects were funded by the EU. Focusing on individual endeavors, the most frequently mentioned project is the EPOCH project, followed by Ariadne and Europeana as well as 3D-COFORM. Since most of the mentioned projects were named by only few researchers, these findings are relatively weak.

\footnotetext{
${ }^{14}$ Since UNESCO Charter are usually named after the place where enacted, search string was: "Charter of."
} 


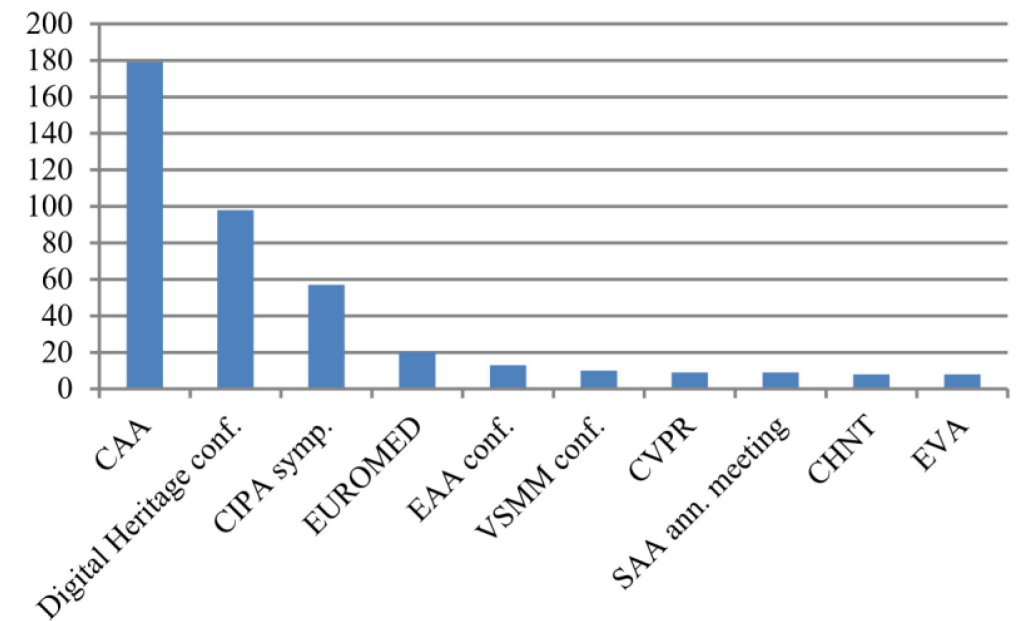

Fig. 10. Conference series named as "most relevant" (Online Survey A, Top 10, $n=411$ out of 1,382 named publications).

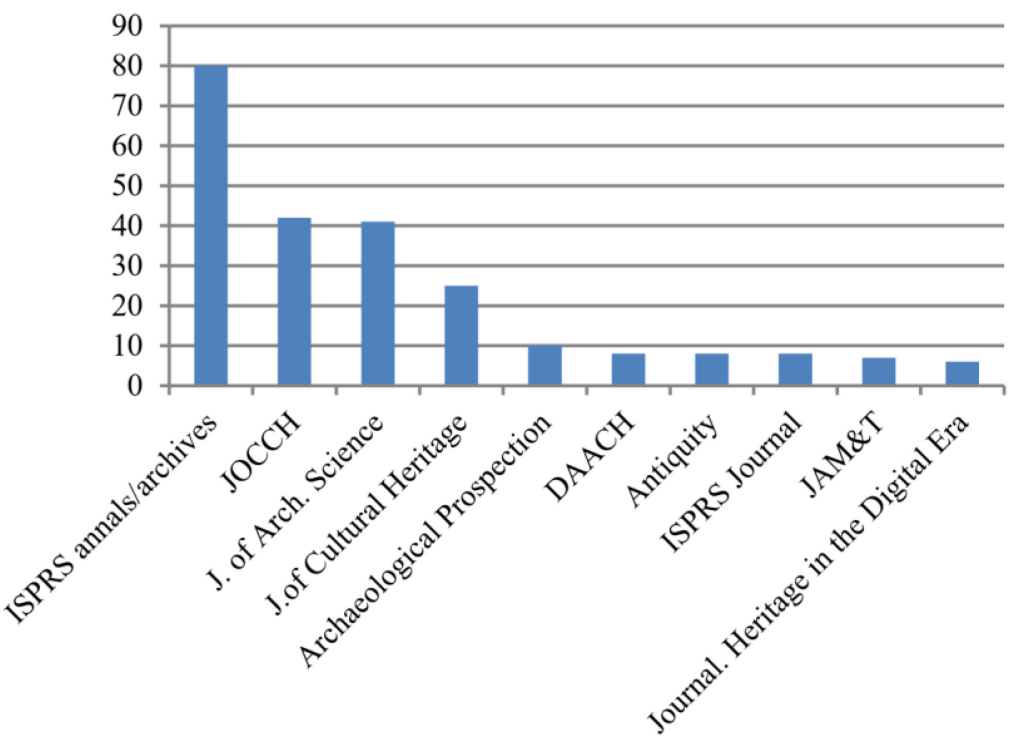

Fig. 11. Journals named as "most relevant" (Online Survey A, Top 10, $\mathrm{n}=235$ out of 1,382 named publications).

How do these findings correlate with quotations in publications? While this ranking widely corresponds to findings of the survey for top positions (Table 10), two major exceptions are the highly referred Europeana and CIDOC-CRM endeavors. A possible explanation may be that their nature is more infrastructure-based (Europeana) or metadata standard-based (CIDOC-CRM) than project-based. ${ }^{16}$

\footnotetext{
${ }^{15}$ Full text retrieval was usually performed non case-sensitive, expect for dictionary terms as CHESS, ARCHES, or EPOCH.

${ }^{16}$ Projects are marked by task-orientation, temporal limitation and transition as well as by teamwork [79] C. Pilbeam, Coordinating temporary organizations in international development through social and temporal embeddedness. International fournal of Project Management, 31, 2 (2013), 190-199; [80] R. A. Lundin and A. Söderholm, A theory of the temporary organization. Scandinavian fournal of Management, 11, 4 (1995), 437-455.
} 


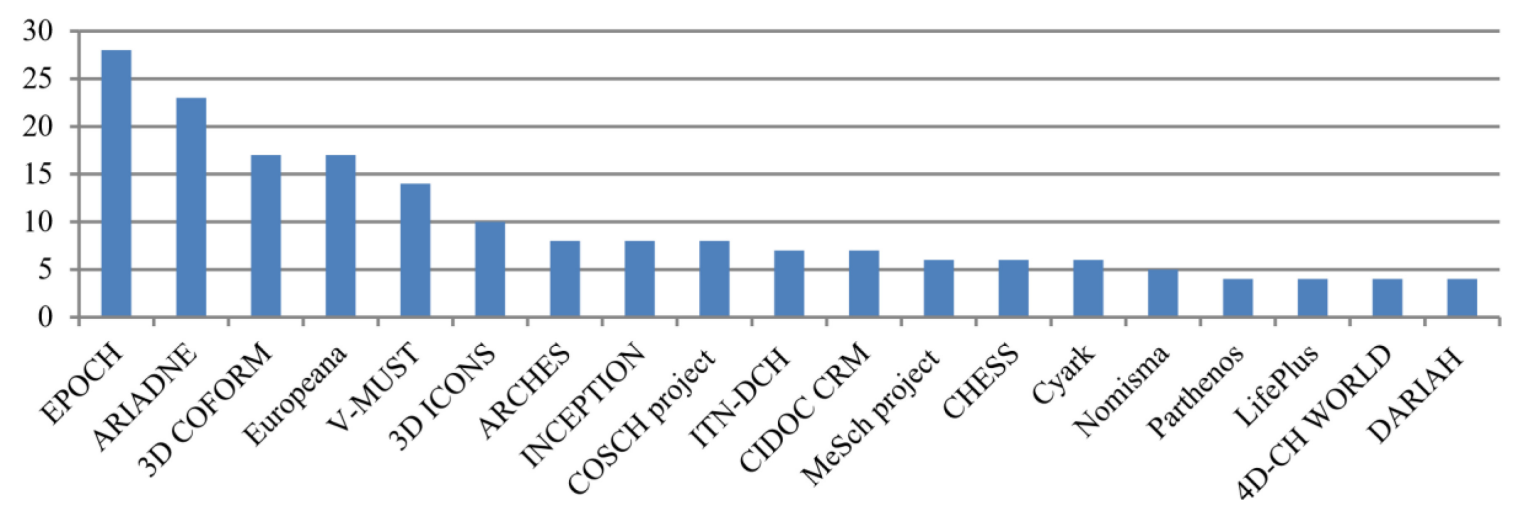

Fig. 12. Projects named as "most relevant" (Online Survey A, N>3, $n=186$ out of 1,024 named projects).

Table 10. Quotation of Selected Projects in Publication Sample B ${ }^{15}$

\begin{tabular}{|l|c|c|c|}
\hline Project & References & Rank in Survey & Rank in Publications \\
\hline EPOCH & 72 & 1 & 3 \\
\hline ARIADNE & 41 & 2 & 4 \\
\hline 3D COFORM & 39 & 3 & 5 \\
\hline Europeana & 124 & 4 & 2 \\
\hline V-MUST & 26 & 5 & 6 \\
\hline 3D ICONS & 17 & 6 & 8 \\
\hline ARCHES & 5 & 7 & 18 \\
\hline INCEPTION & 9 & 8 & 11 \\
\hline COSCH Project & 10 & 9 & 10 \\
\hline ITN-DCH & 6 & 10 & 17 \\
\hline CIDOC CRM & 148 & 11 & 1 \\
\hline MeSch Project & 7 & 12 & 15 \\
\hline CHESS & 9 & 13 & 11 \\
\hline Cyark & 18 & 14 & 13 \\
\hline Nomisma & 8 & 15 & 19 \\
\hline Parthenos & 2 & 16 & 15 \\
\hline LifePlus & 7 & 17 & 17 \\
\hline 4D-CH WORLD & 6 & 18 & 9 \\
\hline DARIAH & 14 & 19 & \\
\hline
\end{tabular}

Against the background that most renowned projects were funded by the EU, another interesting point was if there are other funding sources. I tested this hypothesis by manually assessing "Acknowledgments" of 364 publications in sample B. Regarding a count of projects, most of them were funded by national funding bodies such as ministries, scientific academies or associations (cf. Figure 13). Vice versa, EU projects are often larger than those financed by other funding bodies. As far as it could be identified, a focus of related funding schemes is on scientific and technological development. Topics such as education, cultural aspects like conservation or crossnational cooperation were only occasionally named.

What are main obstacles for employing digital approaches? Another objective of these investigations was to gain an overview of current challenges and demands of stakeholders in the field of digital heritage. These questions were addressed by online survey B and queried in autumn 2017 (cf. Reference [57]). 


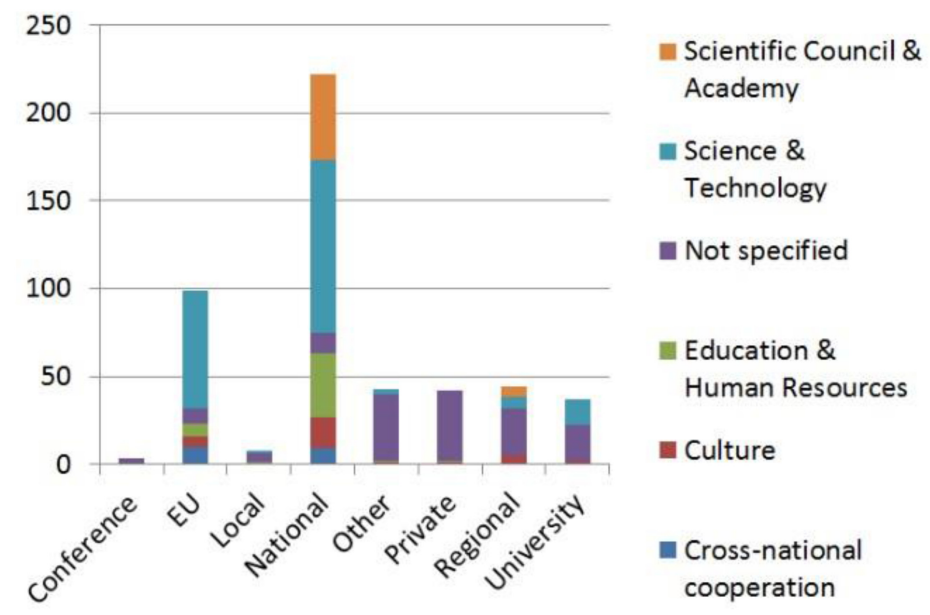

Fig. 13. Funding institutions and programs (Publication Sample B, $n=364$ publications).

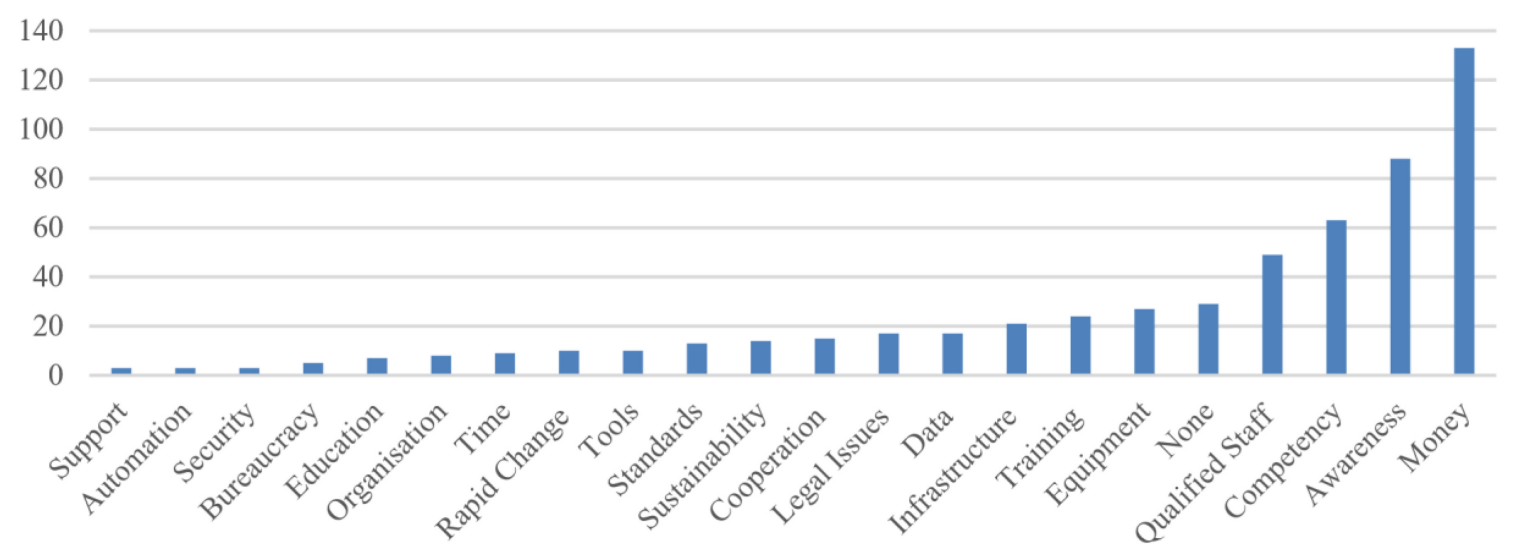

Fig. 14. What would you estimate as main obstacles for employing digital approaches in your organisation? (Online Survey $B, n=264$ items).

Concerning the question of current challenges, money issues were named as biggest obstacle (Figure 14). This comprises lacking funding opportunities for digital activities, costs for hardware and software as well as budget priorities for non-digital activities within organizations. Many participants estimate a missing awareness as a big obstacle. Issues mentioned by interviewees are, for instance, a generation gap in terms of digital literacy and frequency of use of digital tools as well as a general fear of or resistance to digital methods or-vice versamissing awareness of limitations and requirements in the digital world. Finally, a lacking vision of utilizing digital approaches as well as institutional conservatism and tradition were named as hindrance. Concerning human resources, the lack of competency and skills especially in technical domains was frequently named. This closely relates to the frequently mentioned problem of hiring qualified staff skilled in cross-disciplinary cooperation and/or computing. Finally, also training opportunities and resources were named as lacking yet. Vice versa, only one participant named missing employment opportunities for qualified staff. Several participants saw no obstacles for employing digital approaches in their organization. 
What would support a further development?

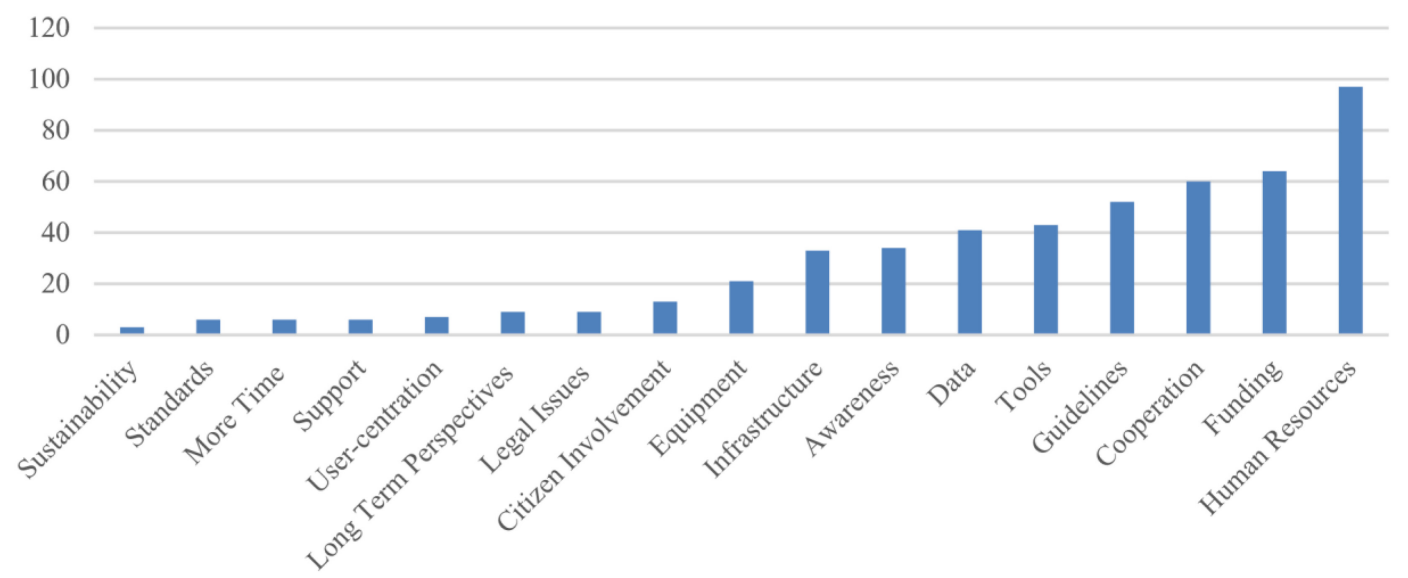

Fig. 15. When developing digital opportunities for your institution and cultural heritage: What would be most helpful for your work? (Online Survey B, $\mathrm{n}=560$ named items).

Concerning demands for a further development (cf. Figure 15), Human Resources were named most frequently, including the need for qualified staff and education. Amongst these aspects, technical expertise and training to increase technical competences were most frequently requested. Many of the participants also requested an increase of student education and involvement of younger people. Specifically suggested were, e.g., coaching opportunities, instruments for knowledge exchange and open training resources, as well as the cross-disciplinary educated staff. At second position, many participants would wish for more funding for staff, equipment, and projects. Occasionally requested were opportunities for small enterprises, non-EU countries as well as for implementation, infrastructures, and long-term availability. Some participants asked for easily accessible funding schemes. At a third position, many participants mentioned an increasing cooperation amongst researchers from different disciplines and/or nations as important. Specific concepts named were to increase intensity and sustainability of cooperation beyond single projects as well as to build specific networks and interest groups on particular topics. The request for guidelines at rank four includes primarily a collection and overview on best practice examples. Also frequently mentioned is the necessity to shape standards and workflows. An emphasis for both aspects is on easy application and user-centred design. Participants also occasionally named documentation and the need for clear objectives as well as roadmaps for further development. Concerning rank five, many participants require software and hardware tools. Beside software for specific purposes, especially open source and easy-to-use tools were demanded. Regarding data listed at rank six, availability, accessibility, sharing and linking were most frequently demanded. Many respondents wish for open access to data. Other frequently mentioned issues are higher data qualities and data validation as well as long-term perspectives and sustainability.

\section{CONCLUSIONS}

What are findings of relevance from these investigations? Regarding a community perspective, most of the researchers in the field of cultural heritage are Europeans and have a disciplinary background in the humanities and, in particular, in archaeology, followed by computing. Even if digital heritage is a relatively new subject and a still emergent community, I identified several facilitators on a structural level. These are individuals with a high number of publications and connections to co-authors, who are important in linking groups of researchers to each other. In comparison to other community members, these facilitators mostly have a strong international network, 
have been involved in academic discourse for many years, and often play key roles in the community in other ways as, for instance, by organizing conferences or heading scholarly associations. The majority of facilitators have been active in the community for no longer than 15 years and currently form the second generation since the 1990s. Cross-national co-authorships are promoted by cultural and spatial closeness and-probably due to funding policy-EU membership.

Even if there is a wide scope of topics addressed, a discourse is primarily driven by technologies, and the most common keywords refer to the technologies used. Most research centers around data in terms of data acquisition and management, visualization or analysis. Moreover, the observed scientific discourse closely relates to practical work in terms of projects relating to specific cultural objects, technologies or practices and is primarily defined by publication bodies and conference series such as CAA or Digital Heritage. Both indications lead to the assumption that the observed scientific community is foremost a community of practice (cf. Reference [14]).

Concerning current challenges, several participants see no obstacles for employing digital approaches in their organization or refer to already well-established digital workflows. Beside that rather small group, money is the biggest obstacle for many researchers; including lacking funding opportunities for digital activities, costs for hardware and software as well as budget priorities for non-digital activities within organizations. Another big problem is a missing awareness due to among others a generation gap or digital divide in terms of digital literacy and frequency of use of digital tools as well as a general fear of or resistance to digital methods or-vice versamissing awareness of limitations and requirements in the digital world. Because the use of digital technologies became common, for instance, in archaeology, it may need to be investigated whether this is a problem affecting the whole field of digital heritage in the same way. Moreover, the lack of competency especially in technical domains is frequently named and vice versa would be seen as most important prospect.

What marks a disciplinary culture of digital heritage? For cultural heritage, digital technologies and approaches are currently considered to linger somewhere between forming another subdomain of humanities and redefining traditional cultural heritage scholarship through digital approaches. Similar to digital humanities, also cultural heritage could be seen as a mode 2 research with an emphasis on cross-disciplinary teamwork, the use of machines and a joint intellectual property. ${ }^{17}$ Consequently, a disciplinary culture on that type of research may be more common to engineering than to humanities. ${ }^{18}$

\subsection{Limitations}

Some potential weaknesses of these investigations have to be mentioned. Due to its focus on international conferences and online publications these investigations may over-represent a specific strand-tangible cultural heritage-but widely exclude other fields, such as intangible cultural heritage or digital born cultural heritage, which may be discussed primarily on smaller conferences instead. Despite publications included in the sample were selected upon feedback from expert interviews and workshops as well as intensively discussed at various conferences, it may be biased. For instance, conference series from the field of museum studies are missing yet. More generally, a focus on academic publications may exclude-as shown in 4.3-institutions like museums, associations or companies. Consequently, especially the US, where those institutions are major players, may be underrepresented. Also, other key trends and technologies as gamification, new media art or the social media

\footnotetext{
${ }^{17}$ The concept of mode 2 research was originally named by Gibbons et al. in 1994 (cf. [81] H. Nowotny, P. Scott, and M. Gibbons, Introduction. Minerva 41 (2003), 179-194; [82] L. K. Hessels and H. V. Lente, Re-thinking new knowledge production: A literature review and a research agenda. Utrecht University, Utrecht, 2007). Some of the attributes named here were initially reported by [83] D. De Solla Price, Little Science-Big Science. Columbia University Press, New York, 1963.

${ }^{18}$ The "solo" scholarship got reflected in several studies about humanities research: [84] L. M. Given and R. Willson, Information Technology and the Humanities Scholar: Documenting Digital Research Practices. Fournal of the Association for Information Science and Technology (2018); [85] E. G. Toms and H. L. O’Brien, Understanding the information and communication technology needs of the e-humanist. fournal of Documentation 64 (2008), 102-130.
} 
are missing yet. Furthermore, the classification of the qualitative answers may be biased-even if detailed coding schemes as the Frascati manual for disciplinary domains [62] were adopted. The quantitative results cannot properly answer the how and why questions. The nomination of EPOCH as most important project, for instance, may be caused by the fact that it was the only big project at that time. Also, the issue of (missing) awareness may not only relate to digital methods but tell about conflicts between generations concerning leadership in that field.

\subsection{Future Research}

Consequently, a future task will be to compare results from the investigations described in this article with those of literature-based investigations and to further assess phenomena, such as, compared to previous investigations, the low rate of researchers with a background in computing. Moreover, it seems of particular interest to repeat the survey periodically to study how the field of research will change over time. With regards to disciplines, recent investigations retrieved by employing language analysis, expert classification, and citation analysis methods revealed trends concerning an increasing or decreasing proximity between various disciplinary domains related to terminology or publication references [86]. Since digital heritage is a highly multi- and cross-disciplinary scholarly field, it would be of interest for me, to what extent original disciplines of authors and survey participants may influence their citation and wording habits. Regarding the scholarly community around digital heritage, it would be especially interesting to further investigate whether my research focus on international conferences and publications in English resulted in a bias toward technology-driven parts of the community. A discourse on specific humanities aspects may be taking place in non-included publications, such as monographs or smaller workshops. Due to the very limited number of journal articles included in this investigation, a closer look on differences and interconnections between journal publications has not been performed yet but might be interesting for a more extended investigation. Since various novel scholarly fields have emerged during the past decades, a comparison to disciplines of similar maturity, such as, for example, the digital humanities, Computer graphics, or Robotics, may also be of interest for future work.

\section{REFERENCES}

[1] UNESCO. 2003. Charter on the Preservation of Digital Heritage. UNESCO, Paris.

[2] S. Schreibman, R. Siemens, and J. Unsworth. 2013. A Companion to Digital Humanities. Blackwell, Oxford.

[3] D. J. Waters. 2013. An overview of the digital humanities. Res. Library Iss. 284 (2013), 3-22.

[4] F. W. Gibbs. 2011. Digital Humanities Definitions by Type. Taylor \& Francis, Milton Park.

[5] S. Münster, F. Rinaudo, R. Tamborrino, F. Apollonio, and M. Ioannides. 2018. Digital Humanities meets Digital Cultural Heritage. In Proceedings of the ADHO DH Conference 2018, Mexico City.

[6] J. Nyhan and A. Flinn. 2016. Computation and the Humanities. Towards an Oral History of Digital Humanities. SpringerOpen, Heidelberg.

[7] M.-C. Tang, Y. J. Cheng, and K. H. Chen. 2017. A longitudinal study of intellectual cohesion in digital humanities using bibliometric analyses. Scientometrics 113, 2 (Nov. 01 2017), 985-1008.

[8] S. Münster and M. Terras. 2019. The visual side of digital humanities. A survey on topics, researchers and epistemic cultures in visual digital humanities. Digital Scholarship in the Humanities (2019).

[9] S. Münster, W. Hegel, and C. Kröber. 2016. A Classification Model for Digital Reconstruction in Context of Humanities Research. Springer LNCS, Cham, 2016.

[10] A. Georgopoulos. 2018. CIPA's Perspectives on Cultural Heritage. Springer, Cham.

[11] K. Knorr-Cetina. 1999. Epistemic Cultures. How the Sciences Make Knowledge. Harvard University Press, Cambridge, 1999.

[12] K. K. Cetina and W. Reichmann. 2015. Epistemic Cultures. Elsevier, Amsterdam.

[13] A. Krishnan. 2009. What are Academic Disciplines. Some Observations on the Disciplinarity vs. Interdisciplinarity Debate? University of Southampton. National Centre for Research Methods, Southhampton.

[14] J. Lave and E. Wenger. 1991. Situated Learning: Legitimate Peripheral Participation. Cambridge University Press, Cambridge.

[15] E. Wenger. 1998. Communities of Practice: Learning, Meaning, and Identity. Cambridge University Press, Cambridge.

[16] S. Münster and M. Ioannides. 2015. The Scientific Community of Digital Heritage in Time and Space. IEEE, Granada.

[17] J. A. Nederhof. 2006. Bibliometric monitoring of research performance in the Social Sciences and the Humanities: A Review. Scientometrics 66, 1 (2006), 81-100.

[18] D. Hicks. 2006. The Four Literatures of Social Science. Springer Science and Business Media, Heidelberg. 
[19] S. Scagliola, B. Safradin, A. Akdag, and S. Wyatt. Mapping Digital Humanities projects, Rotterdam.

[20] L. Börjesson. 2015. Grey literature-Grey sources? Nuancing the view on professional documentation. F. Document. 71, 6 (2015), 11581182.

[21] C. Gumpenberger, J. Sorz, M. Wieland, and J. Gorraiz. 2016. Humanities and social sciences in the bibliometric spotlight - research output analysis at the university of vienna and considerations for increasing visibility. Res. Eval. July 11, 2016.

[22] T. C. E. Engels, T. L. B. Ossenblok, and E. H. J. Spruyt. 2012. Changing publication patterns in the social sciences and humanities, 2000-2009. Scientometrics 93, 2 (2012), 373-390.

[23] L. Leydesdorff, B. Hammarfelt, and A. Salah. 2011. The structure of the arts and humanities citation index: A mapping on the basis of aggregated citations among 1,157 journals. f. Amer. Soc. Info. Sci. Technol. 62, 12 (2011), 2414-2426.

[24] M. Ž. Fuchs. 2014. Bibliometrics: Use and Abuse in the Humanities. Portland Press Limited, Portland.

[25] M. Terras. 2006. Disciplined: Using curriculum studies to define 'humanities computing. Lit. Linguist. Comput. 21, 2 (2006), $229-246$.

[26] S. Weingart. 2016. Submissions to DH2017. 2016.

[27] I. Scollar. 1997. 25 Years of Computer Applications in Archaeology. CAA.

[28] European Commission. 2011. Survey and Outcomes of Cultural Heritage Research Projects Supported in the Context of EU Environmental Research Programmes. From 5th to 7th Framework Programme. European Commission, Brussels.

[29] P. Koutsabasis. 2017. Empirical evaluations of interactive systems in cultural heritage: A review. Int. f. Comput. Methods Heritage Sci. 1 (2017), 100-122.

[30] L. Ciolfi, A. Damala, E. Hornecker, M. Lechner, and L. Maye. 2017. Cultural Heritage Communities: Technologies and Challenges. Routledge.

[31] A. Benardou, E. Champion, C. Dallas, and L. Hughes. 2018. Cultural Heritage Infrastructures in Digital Humanities. Routledge, London.

[32] D. Arnold and G. Geser. 2008. EPOCH Research Agenda-Final Report, Brighton.

[33] J. Drucker. 2013. Is there a "digital" art history? Visual Resour. 29, 1-2 (2013), 5-13.

[34] L. Heusinger. 1989. Applications of Computers in the History of Art. Mansell Pub., London and New York.

[35] H. Kohle. 2013. Digitale Bildwissenschaft. Glückstadt.

[36] T. L. Evans and P. T. Daly. 2006. Digital Archaeology. Bridging Method and Theory. Routledge, London.

[37] E. C. Kansa, S. W. Kansa, and E. Watrall. 2011. ARCHAEOLOGY 2.0. New Approaches to Communication \& Collaboration. Cotsen Digital Archaeology, Los Angeles.

[38] B. Frischer and A. Dakouri-Hild. 2008. Beyond Illustration. 2D and 3D Digital Technologies as Tools for Discovery in Archaeology. Archaeopress, Oxford.

[39] R. Beacham, H. Denard, and F. Niccolucci. 2006. An Introduction to the London Charter.

[40] V. M. L.-M. Bendicho. 2011. The Principles of the Seville Charter.

[41] A. Sürül, H. Òzen, and M. Tutkun. 2003. ICOMOS digital database of the cultural heritage of Trabzon. In Proceedings of the 29th CIPA Symposium, Antalya, Turkey. https://goobi.tib.eu/viewer/image/856489115/611/.

[42] A. Kiouss, M. Karoglou, K. Labropoulos, A. Moropoulou, and R. Zarnic. 2011. Recommendations and Strategies for the Establishment of a Guideline for Monument Documentation Harmonized with the existing European Standards and Codes.

[43] M. Pfarr. 2009. Dokumentationssystem für Digitale Rekonstruktionen am Beispiel der Grabanlage Zhaoling, Provinz Shaanxi, China (PhD Thesis). Darmstadt.

[44] I. Huvila. 2015. Archives, Libraries and Museums in the Contemporary Society: Perspectives of the Professionals. Berlin.

[45] H. Chu. 2015. Research methods in library and information science: A content analysis. Library Info. Sci. Res. 37, 1 (2015), 36-41.

[46] W. Stock and M. Stock. 2015. Handbook of Information Science. De Gruyter Saur, Berlin/Boston.

[47] S. Münster. 2017. Employing Bibliometric Methods to Identify a Community, Topics and Protagonists of Digital 3D Reconstruction in the Humanities. Wuhan.

[48] F. Havemann. 2013. Methoden der Informetrie. De Gruyter, Berlin.

[49] L. Egghe and R. Rousseau. 1990. Introduction to Informetrics. Quantitative Methods in Library, Documentation and Information Science. Elsevier, Amsterdam.

[50] Thomson Reuter. 2015. Source Publication List for Web of Science Arts and Humanities Citation Index. http://mjl.clarivate.com/cgi-bin/ jrnlst/jlresults.cgi?PC=H.

[51] P. Mayring. 2000. Qualitative content analysis. Forum Qualitative Sozialforschung 1, 2 (2000), Art. 20.

[52] S. Münster. 2017. A survey on topics, researchers and cultures in the field of digital heritage. ISPRS Ann. Photogram. Remote Sens. Spatial Info. Sci. IV-2/W2 (2017), 157-162.

[53] H. A. Mieg and M. Näf. 2005. Experteninterviews, Zürich.

[54] J. Gläser and G. Laudel. 2009. Experteninterviews und Qualitative Inhaltsanalyse als Instrumente Rekonstruierender Untersuchungen. VS Verlag für Sozialwissenschaften, Wiesbaden.

[55] P. Mayring. Qualitative Inhaltsanalyse. Grundlagen und Techniken, Weinheim.

[56] U. Reja, K. L. Manfreda, V. Hlebec, and V. Vehovar. 2003. Open-ended vs. close-ended questions in web questionnaires. Dev. Appl. Statist. 19 (2003), 159-177. 
[57] S. Münster, M. Ioannides, and R. Davies. 2017.International Stakeholder Survey on Demands in the Field of Digital Cultural Heritage. https://www.researchgate.net/publication/321698674_International_stakeholder_survey_on_demands_in_the_field_of_digital_ cultural_heritage.

[58] M. Porter. 1980. An algorithm for suffix stripping. Program 3, 14 (1980), 130-137.

[59] Provalis Research. 2010. WordStat 6. Content Analysis Module for QDA Miner and SimStat. User's Guide. Provalis Research, Montreal.

[60] S. Münster, T. Köhler, and S. Hoppe. 2015. 3D Modeling Technologies as Tools for the Reconstruction and Visualization of Historic Items in Humanities. A Literature-based Survey. Amsterdam University Press, Amsterdam.

[61] S. Münster, P. Kuroczyński, M. Pfarr-Harfst, M. Grellert, and D. Lengyel. 2015. Future research challenges for a computer-based interpretative 3D reconstruction of cultural heritage - A german community's view. Proceedings of the 25th International CIPA Symposium on Photogrammetry, Remote Sensing, and Spatial Information Sciences (ISPRS'15), II-5-W3. 207-213.

[62] Organisation for Economic Co-operation and Development. 2007. Revised Field of Science and Technology (FOS) Classification in the Frascati Manual. Paris.

[63] CERIF (Common European Research Project Information Format). 1991. Offic. J. Eur. Commun. L189 (1991).

[64] NCES IPEDS. 2010. Classification of Instructional Programs (CIP). https://nces.ed.gov/ipeds/cipcode/default.aspx?y=55.

[65] S. Münster. 2016. Interdisziplinäre Kooperation bei der Erstellung Geschichtswissenschaftlicher 3D-Rekonstruktionen. Springer VS, Wiesbaden, 2016.

[66] M. S. Granovetter. 2013. Strength of the weak ties. Amer. F. Sociol. 78, 6 (1973), 1360-1380.

[67] C. Stützer. 2013. Knowledge Transfer in Web-based Collaborative Learning Systems (Ph.D. Thesis), Dresden.

[68] S. Wasserman and K. Faust. 1994. Social Network Analysis. Cambridge University Press, Cambridge.

[69] T. Velden and C. Lagoze. Patterns of collaboration in co-authorship networks in chemistry - mesoscopic analysis and interpretation. In Proceedings of the 12th International Conference of the International Society for Scientometrics and Informetrics (ISSI'09). http://www. issi-society.org/publications/issi-conference-proceedings/proceedings-of-issi-2009/.

[70] T. Velden, A. Haque, and C. Lagoze. 2010. A new approach to analyzing patterns of collaboration in co-authorship networksMesoscopic analysis and interpretation. Scientometrics 85, 1 (2010), 219-242.

[71] A. Schubert and W. Glänzel. 2006. Cross-national preference in co-authorship. Scientometrics 69, 2 (2006), 409-428.

[72] W. Glänzel. 2001. National characteristics in international scientific co-authorship relations. Scientometrics 51, 1 (2001), 69115.

[73] D. D. Beaver. 2001. Reflections on scientific collaboration (and its study): Past, present, and future. Scientometrics 52, 3 (2001), 365-377.

[74] A. J. Lotka. 1926. The frequency distribution of scientific productivity. F. Washington Acad. Sci. 16, 12 (1926), 317-323.

[75] L. Egghe. 2000. A heuristic study of the first-citation distribution. Scientometrics 48, 3 (2000), 345-359.

[76] L. J. Murphy. 1973. "Lotka's law in the humanities?" F. Amer. Soc. Info. Sci. 24, 6 (1973), 461-462.

[77] A. Pulgarín. 2012. Dependence of lotka's law parameters on the scientific area. Malaysian f. Library Info. Sci. 17, 1 (2012), 41-50.

[78] M. P. Long and R. C. Schonfeld. 2014. Supporting the Changing Research Practices of Art Historians. Ithaka S+R, Princeton.

[79] C. Pilbeam. 2013. Coordinating temporary organizations in international development through social and temporal embeddedness. Int. f. Project Manage. 31, 2 (2013), 190-199.

[80] R. A. Lundin and A. Söderholm. 1995. A theory of the temporary organization. Scand. F. Manage. 11, 4 (1995), 437-455.

[81] H. Nowotny, P. Scott, and M. Gibbons. 2003. Introduction. Minerva 41 (2003), 179-194.

[82] L. K. Hessels and H. V. Lente. 2007. Re-thinking new Knowledge Production: A Literature Review and a Research Agenda. Utrecht University, Utrecht.

[83] D. De Solla Price. 1963. Little Science - Big Science. Columbia Univ. Press, New York (1963).

[84] L. M. Given and R. Willson. 2018. Information technology and the humanities scholar: Documenting digital research practices. F. Assoc. Info. Sci. Technol. 69, 6 (2018), 807-819.

[85] E. G. Toms and H. L. O’Brien. 2008. Understanding the information and communication technology needs of the e-humanist. F. Document. 64 (2008), 102-130.

[86] L. Dias, M. Gerlach, J. Scharloth, and E. G. Altmann. 2017. Using text analysis to quantify the similarity and evolution of scientific disciplines. R Soc Open Sci. 17, 5 (2017).

Received June 2018; revised January 2019; accepted January 2019 